\title{
Aminopyridines Correct Early Dysfunction and Delay Neurodegeneration in a Mouse Model of Spinocerebellar Ataxia Type 1
}

\author{
Raphael Hourez, ${ }^{1}$ Laurent Servais,,${ }^{1,3 *}$ David Orduz, ${ }^{1 *}$ David Gall, ${ }^{1 \star}$ Isabelle Millard, ${ }^{2}$ Alban de Kerchove d'Exaerde, ${ }^{1}$ \\ Guy Cheron, ${ }^{3}$ Harry T. Orr, ${ }^{4}$ Massimo Pandolfo, ${ }^{2}$ and Serge N. Schiffmann ${ }^{1}$ \\ ${ }^{1}$ Laboratory of Neurophysiology and ${ }^{2}$ Laboratory of Experimental Neurology, Université Libre de Bruxelles, 1070 Brussels, Belgium, ${ }^{3}$ Laboratory of \\ Electrophysiology, Université de Mons-Hainaut, 7000 Mons, Belgium, and ${ }^{4}$ Department of Genetics, Cell Biology, and Development, University of \\ Minnesota, Minneapolis, Minnesota 55455
}

The contribution of neuronal dysfunction to neurodegeneration is studied in a mouse model of spinocerebellar ataxia type 1 (SCA1) displaying impaired motor performance ahead of loss or atrophy of cerebellar Purkinje cells. Presymptomatic SCA1 mice show a reduction in the firing rate of Purkinje cells (both in vivo and in slices) associated with a reduction in the efficiency of the main glutamatergic synapse onto Purkinje cells and with increased A-type potassium current. The A-type potassium channel Kv4.3 appears to be internalized in response to glutamatergic stimulation in Purkinje cells and accumulates in presymptomatic SCA1 mice. SCA1 mice are treated with aminopyridines, acting as potassium channel blockers to test whether the treatment could improve neuronal dysfunction, motor behavior, and neurodegeneration. In acutely treated young SCA1 mice, aminopyridines normalize the firing rate of Purkinje cells and the motor behavior of the animals. In chronically treated old SCA1 mice, 3,4-diaminopyridine improves the firing rate of Purkinje cells, the motor behavior of the animals, and partially protects against cell atrophy. Chronic treatment with 3,4-diaminopyridine is associated with increased cerebellar levels of BDNF, suggesting that partial protection against atrophy of Purkinje cells is possibly provided by an increased production of growth factors secondary to the reincrease in electrical activity. Our data suggest that aminopyridines might have symptomatic and/or neuroprotective beneficial effects in SCA1, that reduction in the firing rate of Purkinje cells can cause cerebellar ataxia, and that treatment of early neuronal dysfunction is relevant in neurodegenerative disorders such as SCA1.

\section{Introduction}

Some manifestations of neurodegenerative diseases likely reflect alterations in the function of neurons rather than neuronal atrophy and loss. Electrophysiological changes accompanying

Received Feb. 17, 2011; revised May 26, 2011; accepted June 13, 2011.

Author contributions: R.H., L.S., D.G., D.O., I.M., M.P., and S.N.S. designed research; R.H., L.S., D.G., D.O., I.M., A.d.K.d., G.C., M.P., and S.N.S. performed research; H.T.O. contributed unpublished reagents/analytic tools; R.H., L.S., D.G., D.O., I.M., A.d.K.d., M.P., and S.N.S. analyzed data; R.H., L.S., D.G., D.O., I.M., M.P., and S.N.S. wrote the paper.

This work was supported by Fonds National de la Recherche Scientifique (FNRS), European Integrated Project on Spinocerebellar Ataxias (EUROSCA), Fondation Médicale Reine Elisabeth, Van Buuren Foundation, Action de Recherche Concertée, and research funds of Université Libre de Bruxelles (ULB) and Université Mons-Hainaut (UMH). R.H. was supported by FNRS (Aspirant-FNRS, and then Chargé De Recherché FNRS), Belgian Neurological Society, HorlaitDapsens Foundation, and Hereditary Disease Foundation. L.S. and A.d.K.d. were supported by FNRS, and I.M. was supported by EUROSCA. We warmly thank Drs. David Blum and Satyan Chintawar for very helpful discussions, opinions, and insights. The technical expertise of Delphine Houteman and Laetitia Cuvelier was critical for this work and is greatly appreciated. We thank Prof. Parminder Vig (University of Mississippi Medical Center, Jackson, MS) for providing B05 mice and Prof. James Trimmer (University of California, Davis, School of Medicine, Davis, CA) for providing Kv4.2 and Kv4.3 antibodies.

*L.S., D.O., and D.G. contributed equally to this work.

Correspondence should be addressed to Serge N. Schiffmann, Laboratory of Neurophysiology, Université Libre de Bruxelles, Route de Lennik 808, CP601, 1070 Brussels, Belgium. E-mail: sschiffm@ulb.ac.be.

R. Hourez's present address: Centre Hospitalier Universitaire Vaudois (CHUV), Department of Neurosciences (DNC), rue du Bugnon, 1011 Lausanne, Switzerland.

L. Servais's present address: Institut de Myologie, Hôpital de la Pitié Salpétrière, 48/83 Bvd de l'hôpital, 75013 Paris, France.

DOI:10.1523/JNEUROSCI.0905-11.2011

Copyright $\odot 2011$ the authors $\quad 0270-6474 / 11 / 3111795-13 \$ 15.00 / 0$ neuronal dysfunction are not well characterized in all neurodegenerative disorders so it is unclear whether this type of neuronal dysfunction contributes to neuronal atrophy and could be a relevant therapeutic target. In the case of Huntington's disease, it has been suggested that initial stages of the disease result from dysfunction rather than cell death (Tobin and Signer, 2000), and recent electrophysiological studies in presymptomatic YAC mice confirmed this point (Milnerwood et al., 2010). Simultaneous study of behavior and pathology demonstrated that neurological deficits may occur without neuronal loss in models of spinocerebellar ataxia type 1 (SCA1) (Burright et al., 1995; Clark et al., 1997). Neuronal dysfunction is sometimes considered as a compensatory response or as a marker of underlying pathogenic mechanisms in neurodegenerative disorders. However, conditions such as spinocerebellar ataxia type 13 (SCA13), caused by mutations in the gene encoding a potassium channel and resulting in altered neuronal electrical excitability and progressive neuronal loss (Waters et al., 2006), illustrate that neuronal dysfunction can directly contribute to the development of pathology.

We studied an early electrophysiological dysfunction of cerebellar Purkinje cells (PCs) in a mouse model of SCA1, a disorder characterized by progressive cerebellar ataxia and loss of cerebellar PCs and caused by a CAG triplet repeat expansion in the ATX1 gene resulting in an expanded polyglutamine tract in the protein ataxin-1. In this model, human 


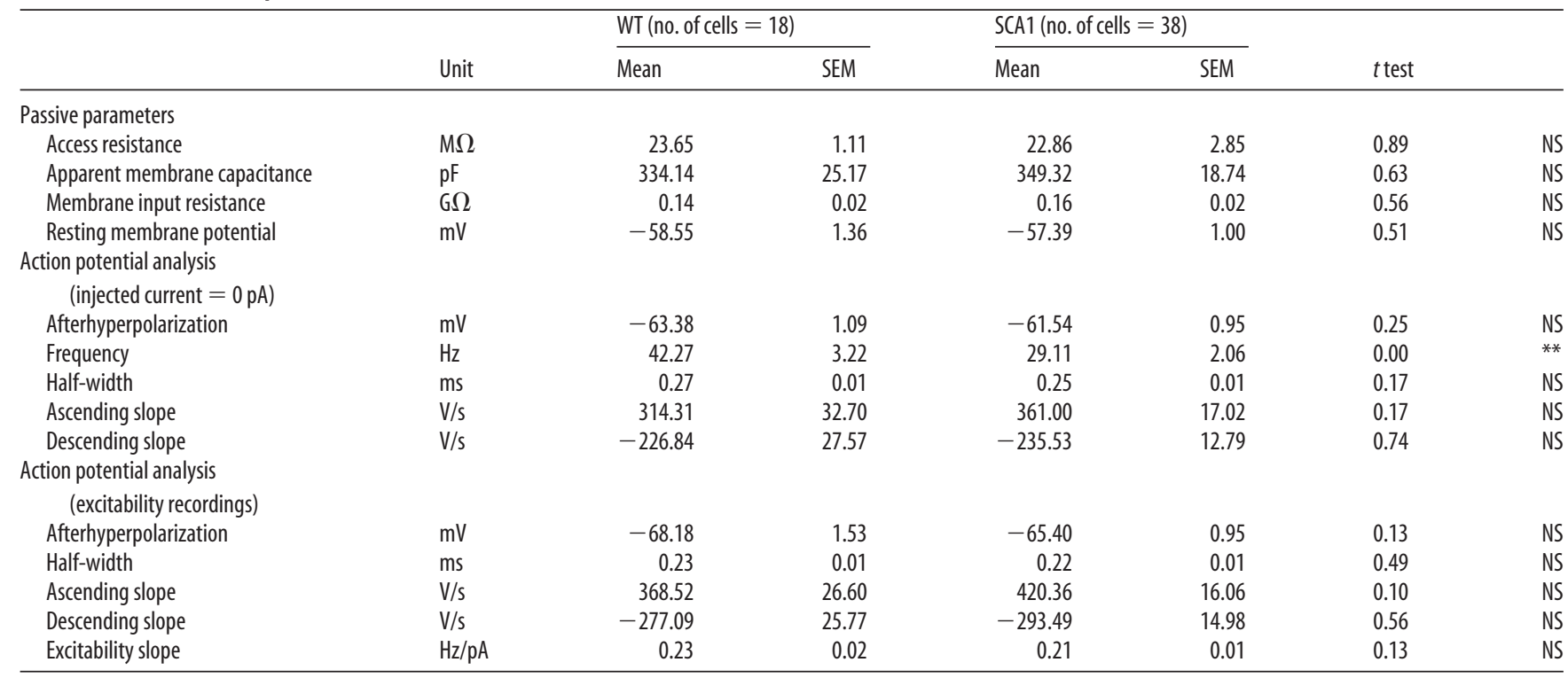

Passive parameters and action potential analysis from patch-clamp recordings of Purkinje cells from 2- to 3-week-old WT and SCA1 mice.

NS, No significant difference $(p>0.05) ;{ }^{* *} p<0.01$.

expanded ataxin-1 is expressed under the control of a promoter specific for PCs (Burright et al., 1995; Clark et al., 1997). The animals develop motor abnormalities before histopathological changes are visible, suggesting a possible early neuronal dysfunction. While previous electrophysiological studies of SCA1 mice were performed at a late stage with major morphological alterations of PCs (Inoue et al., 2001), we focused on anomalies already present in presymptomatic mice and attempted to test whether correction of early neuronal dysfunction of PCs delayed later cell atrophy.

\section{Materials and Methods}

Animal model. A02 and B05 mice express human ataxin-1 with a normal (30) or pathological (82) CAG repeat length specifically within cerebellar PCs using the pcp2 promoter as previously described (Burright et al., 1995; Clark et al., 1997). Animal care was in accordance with European Commission regulations for animal use in research (CEE no. 86/609) and the local ethical committee.

Histology. Histology was performed as previously described (Servais et al., 2007). Antibodies used were rabbit anti-calbindin-D28k (1/500; Swant), goat polyclonal Kv4.1 antibody (1/50; Santa Cruz Biotechnology), or mouse monoclonal Kv4.2 antibody $(10 \mu \mathrm{g} / \mathrm{ml})$ or mouse monoclonal Kv4.3 antibody $(100 \mu \mathrm{g} / \mathrm{ml})$ (both gifts from James S. Trimmer, University of California, Davis, Davis, CA). PCs were counted in cerebellar lobule $\mathrm{X}$ in three successive mediosagittal slices per mouse from five different mice per group. Thickness of the molecular layer was assessed as previously described ( $\mathrm{Zu}$ et al., 2004). Thickness of the molecular layer and PC counts were assessed by an investigator blind for genotype and treatment group.

Motor coordination assessment. Accelerating rotarod (Ugo Basile) was performed as previously described (Servais et al., 2007). Tested groups were weight matched. The grip strength test (Bioseb) was used to test forelimb grip strength (the task also requires motor coordination). The stationary horizontal thin rod test was done as described previously (Kashiwabuchi et al., 1995). Motor behavior assessment was performed by two investigators blind for both genotype and treatment.

Electrophysiology. Slice preparation, perforated-patch recordings, and study of the parallel fiber-Purkinje cell synapse by electrical stimulation of parallel fibers were performed as previously described (Servais et al., 2007). Slices were perfused with an oxygenated extracellular solution containing the following: $120 \mathrm{~mm} \mathrm{NaCl}, 26 \mathrm{~mm} \mathrm{NaHCO}_{3}, 2 \mathrm{~mm} \mathrm{KCl}, 2$ $\mathrm{mM} \mathrm{CaCl}_{2}, 1.19 \mathrm{~mm} \mathrm{MgSO}_{4}, 1.18 \mathrm{~mm} \mathrm{NaH}_{2} \mathrm{PO}_{4}$, and $11 \mathrm{~mm}$ glucose at room temperature $\left(20-25^{\circ} \mathrm{C}\right)$. Picrotoxin $(100 \mu \mathrm{M})$ was added to the extracellular solution for the recording of evoked or spontaneous EPSCs. Measurement of action potential parameters were performed in two distinct conditions: from the resting membrane potential, in the absence of injected current (Table 1, "injected current $=0 \mathrm{pA}^{\text {") }}$ or when first appearing during "excitability recordings" (Table 1). In the latter recordings, membrane potential was clamped to $-80 \mathrm{mV}$ and depolarizing currents of $50 \mathrm{pA}$ increments were applied. Measurement of PC firing frequency after pharmacological treatment of the mice was performed in the hour following the sacrifice of the animal to limit washout effects. For mEPSC recordings, patch pipettes (3-5 M $\Omega$ ) contained the following (in mM): $110 \mathrm{CsSO}_{4}, 10$ HEPES, 10 EGTA, $1.5 \mathrm{MgCl}_{2}, 0.4$ GTP-Na, 4 ATP$\mathrm{Na}$; TTX $1 \mu \mathrm{M}$ was added to the extracellular solution. Recordings were performed in whole-cell configuration, and holding potential was maintained at $-70 \mathrm{mV}$. mEPSCs were filtered at $2 \mathrm{kHz}$ and digitally sampled at $20 \mathrm{kHz}$; the sampling duration was $300-1000 \mathrm{~s} /$ cell. mEPSCs were analyzed with Mini Analysis software (Synaptosoft), detection parameters (amplitude, area, decay, and peak times) were determined from hand-picked minievents before automatic detection, each detected mEPSC was verified visually. More than 2000 events were obtained from the WT and SCA1 groups. Recordings of $\mathrm{IK}_{\mathrm{A}}$ were made as described previously (Kim et al., 2007). The A-type potassium current was isolated by the subtraction of potassium currents evoked by a depolarization to $-20 \mathrm{mV}$ after hyperpolarized $(-80 \mathrm{mV})$ and depolarized $(-40 \mathrm{mV})$ conditioning steps $(200 \mathrm{~ms})$ from a holding potential of $-70 \mathrm{mV}$, in presence of TEA (tetraethylammonium). rHongotoxin-1, stromatoxin (Alomone) were added to the extracellular solution at the final concentration of $0.1 \mu \mathrm{M}$. Phrixotoxin-2 (Alomone) was added at the final concentration of $1 \mu \mathrm{M}$. Each potassium channel blocker was applied to two to three cells from both WT and SCA1 mice. After recording IK $\mathrm{A}_{\mathrm{A}}$, glutamate

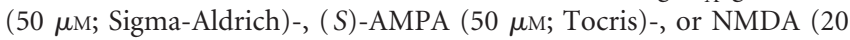
$\mu \mathrm{M}$; Tocris)-containing external solution was applied and currents were recorded every $5 \mathrm{~min}$ for $15 \mathrm{~min}$. All recordings and analysis of firing frequency of PCs were made in blind for the genotype or treatment group.

Biocytin injection for PC morphology and cell volume quantification. In a separate series of whole-cell recordings used for morphological reconstruction, the intracellular solution contained the following (in $\mathrm{mM}$ ): 126 K-gluconate, $0.05 \mathrm{CaCl}_{2}, 0.15 \mathrm{BAPTA}, 4 \mathrm{NaCl}, 1 \mathrm{MgSO}_{4}, 15$ glucose, 5 HEPES, 3 MgATP, 0.1 GTP, and $0.4 \%$ biocytin (Sigma-Aldrich). PCs were filled with biocytin during $15 \mathrm{~min}$, and fluorescence was subsequently revealed by cytochemistry. Slices were fixed by immersion in $4 \%$ paraformaldehyde overnight. Biocytin was revealed with streptavidin- 

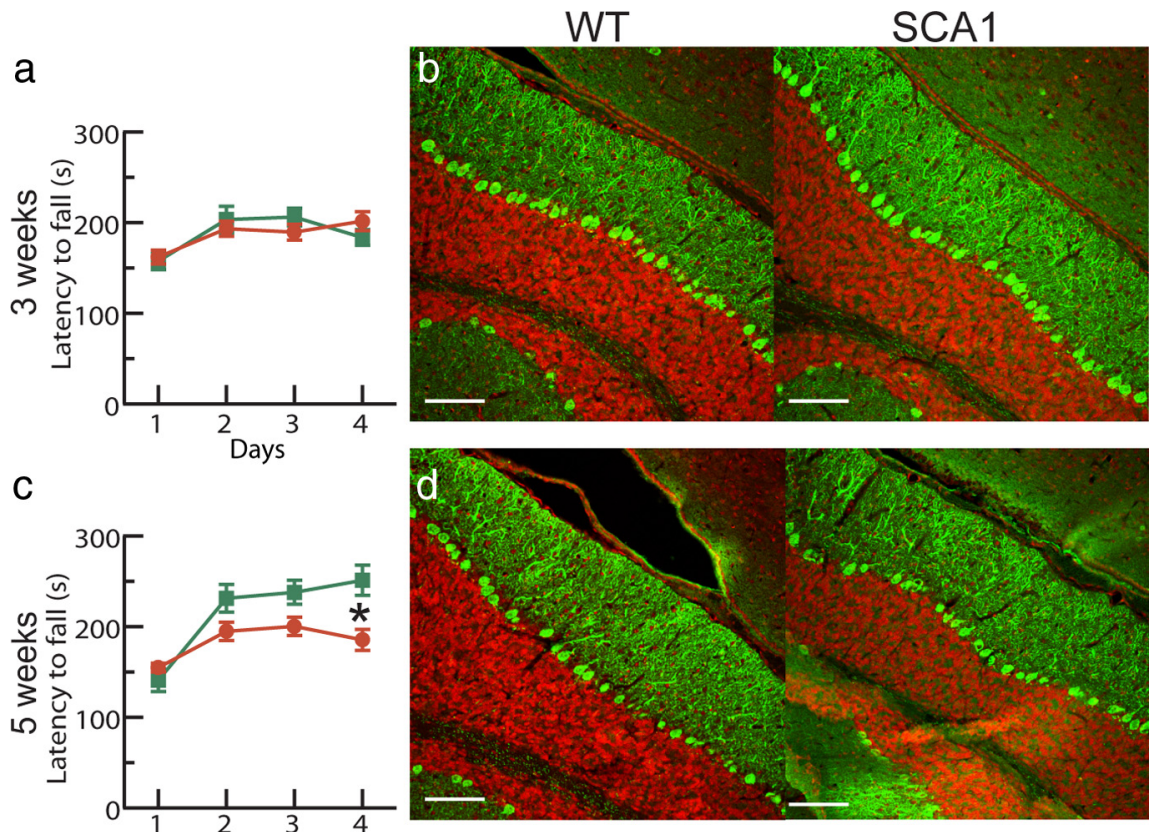

e
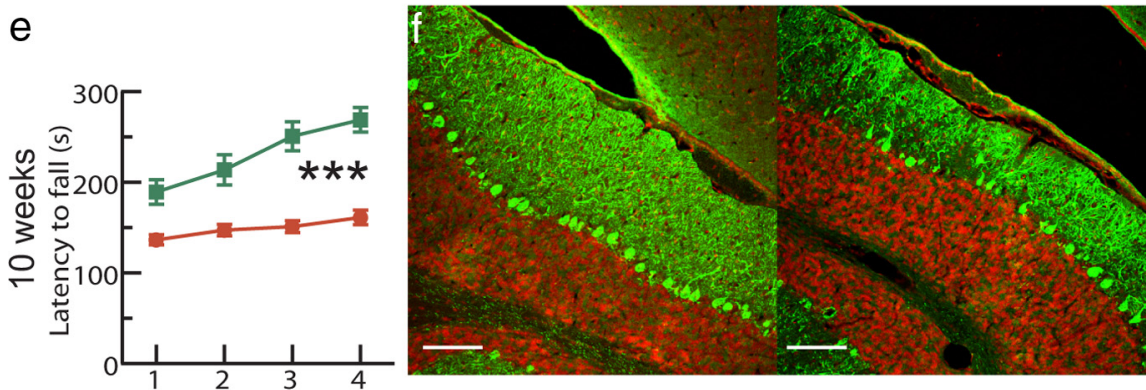

g
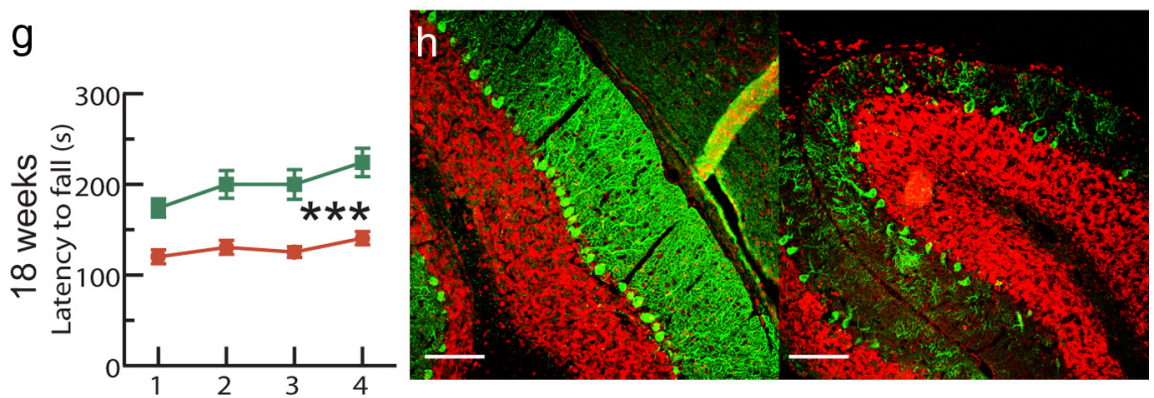

Figure 1. Time courses of motor behavior, morphological alterations, and cell death in SCA1 mice. Motor coordination was assessed by the latency to fall from an accelerated rotarod; morphology was assessed by calbindin labeling in green (toto red counterstaining). Scale bar, $60 \mu \mathrm{m}$. Three-week-old SCA1 mice have normal motor coordination (a) and normal morphology (b). Five-week-old SCA1 mice display impaired motor performance (c), but no morphological alteration (d). Ten-week-old SCA1 mice display impaired motor performance $(\boldsymbol{e})$ and morphological alterations $(\boldsymbol{f})$. Eighteen-week-old SCA1 mice display impaired motor performance $(\boldsymbol{g})$ and morphological alterations $(\boldsymbol{h})$. Rotarods: two-way ANOVA followed by Fisher's test, 3 weeks, 13 WT and 16 SCA1, $p=0.92 ; 5$ weeks, 14 WT and 14 SCA1, $p=0.043 ; 10$ weeks, 14 WT and 14 SCA1, $p=1.2 \times 10^{-5} ; 18$ weeks, 20 WT and 19 SCA1, $p=5.2 \times 10^{-5} .{ }^{*} p<0.05 ;{ }^{* * *} p<0.001$. Error bars indicate SEM in all figures

conjugated fluorescein isothiocyanate 1:200 (FITC; Jackson ImmunoResearch). Images were acquired using a LSM 510 META laser-scanning confocal system (Zeiss) mounted on an Axiovert 200M inverted microscope (Zeiss) equipped with c-Achroplan NIR $40 \times / 0.8 \mathrm{~W}$ objective (Zeiss). The excitation beam of an argon laser $(488 \mathrm{~nm})$ and bandpass emission filters $(500-550 \mathrm{~nm})$ were used for selective detection of the green fluorochrome. Sequential optical sections of $2048 \times 2048$ pixels were taken at $1.0 \mu \mathrm{m}$ intervals along the $z$-axis to allow 3D reconstruction. Cellular volumes were computed by evaluating the number of voxels inside cells. For each cell, the threshold value defining the cell surface was set using the ISODATA algorithm implemented in NIH Image soft- ware. The total number of voxels inside the cell was then evaluated using the VoxelCounter plug-in (NIH) for ImageJ and multiplied by the unitary voxel volume. Dendritic spines numbers per unit length were obtained by visually counting the spines on $z$-projections and dividing by the length of dendrites. To avoid any bias, data acquisition and analysis were performed in the absence of knowledge of the genotype or treatment group.

In vivo extracellular Purkinje cell recordings and eyelid conditioning. In vivo extracellular Purkinje cell recordings and eyelid conditioning in alert mice were performed as previously described (Servais et al., 2007). Briefly, the day before recording sessions, mice were anesthetized with xylido-dihydrothiazin (Rompun; 10 $\mathrm{mg} / \mathrm{kg}$; Bayer) and ketamine (Ketalar; $100 \mathrm{mg} /$ $\mathrm{kg}$; Pfizer). In addition, local anesthesia with $0.5 \mathrm{ml}$ of lidocaine $(20 \mathrm{mg} / \mathrm{ml})$ plus adrenaline (1:80,000) (xylocaine; AstraZeneca) was administered subcutaneously during soft tissue removal. Two small bolts were cemented to the skull to immobilize the head during the recording sessions, and a silver reference electrode was placed on the surface of the parietal cortex. An acrylic recording chamber was constructed around a posterior craniotomy, covered by a thin layer of bone wax (Ethicon; Johnson \& Johnson). Twenty-four hours after anesthesia, alert mice were immobilized in a recording stage, the dura mater was removed locally above the vermis, and recordings were performed in lobules IV-VIII with glass micropipettes filled with $0.2 \mathrm{M} \mathrm{NaCl}$ (1.5-5 $\mathrm{M} \Omega$ of impedance). A neural signal was considered as originating from a Purkinje cell if it presented two types of spiking activities: simple spikes characterized by single depolarization (300$800 \mathrm{~ms}$ ) occurring between 20 and $200 \mathrm{~Hz}$ and complex spikes characterized by an initial fast depolarization (300-600 ms), followed by smaller and relatively constant wavelets. It was considered that simple and complex spikes originated from the same Purkinje cell when a transient pause $(\sim 20 \mathrm{~ms})$ in simple spike firing followed each complex spike.

Western blotting. Western blotting was performed as previously described (Servais et al., 2007). Antibodies used were rabbit anti-Kv4.1, Kv4.2, or Kv4.3 (1/200), antiBDNF (1/500) [BDNF (N-20): sc-546; Santa Cruz Biotechnology], mouse anti-actin (1/ 10,000) (Sigma-Aldrich), mouse anti-polyglutamine 1C2 (1/1000) (Millipore Bioscience Research Reagents), or mouse anti-GAPDH (1/ 10,000) (Sigma-Aldrich).

Biotinylation assay. Biotinylation assay was performed as previously described (Beazely et al., 2009) on acute slices of the anterior vermis with anti-Kv4.3 (NeuroMab; 1:1000), anti-Rab4 (BD Biosciences Transduction Laboratories; $1: 1000)$.

In situ hydridization. In situ hydridization was performed as previously described (Schiffmann et al., 1999) using the following probes: Kv4.1, CCC TCG GGA TGC CTT CAC CTC CGG TGC AGG GGG TAG GGG CTG CTG and GCC TGC TGG AAG GGC TGG ACC TTC CCC GGC CTG CTC AGC CTC CTC, Kv4.2, GCT TCC CGA CAC ATT GGC ATT AGG AAT GCG GAA AGT CTT TTT GTG; Kv4.3, TTG CCG CTT GTT CTT GTC CGC TGG AGC TAG GGG CAT GGG 
GCA GTT and GAA GCT GAG AGA GGG CAT GGA CTC CTG GTT GTT TTC AGA GTC ATT.

Laser microdissection. Brains were extracted in RNase-free conditions and quickly frozen in isopentane, and sections were made immediately before microdissection and mounted on PALM MembraneSlides PEN/NF (PALM Microlaser Technologies). Sections were dried at room temperature for 2-3 s before dehydration, coloration with cresyl violet to allow PC visualization and rehydration. PCs were visualized, cut, and catapulted to PALM Adhesive Caps closed with LPC-Microfuge Tubes clear (PALM) and frozen in dry ice. Approximately 2000 cerebellar PCs were microdissected for each animal.

$q R T-P C R$. qRT-PCR was performed using the SYBR Green PCR Master Mix (Applied Biosystems) on a 7500 Fast Real-Time PCR System (Applied Biosystems). Primers were as follows: Kv4.1, forward, 5'-CCT CTG TCA GCC GTG GCA-3', and reverse, 5' -TGA GGC TTG AGC GGG TTT G-3'; Kv4.2, forward, 5' ACA AAC GAA GGG CAC AGA AGA A-3', and reverse, 5' -CAT GTA GGC ATT TGC ACT CCC-3'; Kv4.3, forward, 5'-TGC CAG CAA GTT CAC AAG CA-3', and reverse, 5'-GCA CCA TGT CTC CAT ATC CCA-3'; B2M, forward, 5' -TGC TAT CCA GAA AAC CCC TCA A-3', and reverse, 5'-GAG GCG GGT GGA ACT GTG T-3'; TBP, forward, 5'-TGT ACC GCA GCT TCA AAA TAT TGT AT-3', and reverse, 5'-AAA TCA ACG CAG TTG TCC GTG-3'; RER1, forward, 5'-CCA CCT AAA CCT TTT CAT TGC G- $3^{\prime}$, and reverse, $5^{\prime}$-TTT GTA GCT GCG TGC CAA AAT-3'; RPL13, forward, $5^{\prime}$-CCC GTG GCG ATT GTG AA-3', and reverse, $5^{\prime}$-TCA TTG TCC TTC TGT GCA GGT T. Reporter genes were B2M and TBP except for qRT-PCR performed on the anterior vermis, where RER 1 and RPL13 were used. The relative expression between SCA1 transgenic and wild-type littermate mice was measured using the Pfaffl method modified by Vandesompele et al. (2002) for multiple normalization using geometric mean and analyzed using qBase (http://medgen.ugent.be/qbase/).

Pharmacological treatments. Fresh solutions of 3,4-diaminopyridine (DiAP) $(5 \mathrm{mg} /$ kg; Sigma-Aldrich) and 4-aminopyridine (AP) $(1 \mathrm{mg} / \mathrm{kg}$; Sigma-Aldrich) in $0.9 \% \mathrm{sa}-$ line were injected subcutaneously after the $\mathrm{pH}$ was adjusted to $\mathrm{pH} 7.3-7.5$ with $\mathrm{HCl}$. A 45 min interval was used between injection and behavioral assessment or sacrifice and electrophysiological recordings to insure drug bioavailability. These dosages and delays were well tolerated and the most effective preventing dyskinesia attacks in tottering mice (Weisz et al., 2005). Chronic subcutaneous treatment was performed using $200 \mu \mathrm{l}$ Alzet miniosmotic pumps (model 2004; Durect Corporation) implanted subcutaneously in the back of the mice. The pumps allowed a continuous $0.25 \mu \mathrm{l} / \mathrm{h}$ delivery of the solution for $28 \mathrm{~d}$. Pumps were replaced every $30 \mathrm{~d}$ so that they were treated from 4 to $17-18$ weeks and killed at 18 weeks. The limiting factor was the solubility of 3,4-diaminopyridine, so that $144 \mu \mathrm{g}$ of 3,4-diaminopyridine was delivered a day during the whole chronic treatment. (right) mice. Scale bar, $8 \mu \mathrm{m}$. a
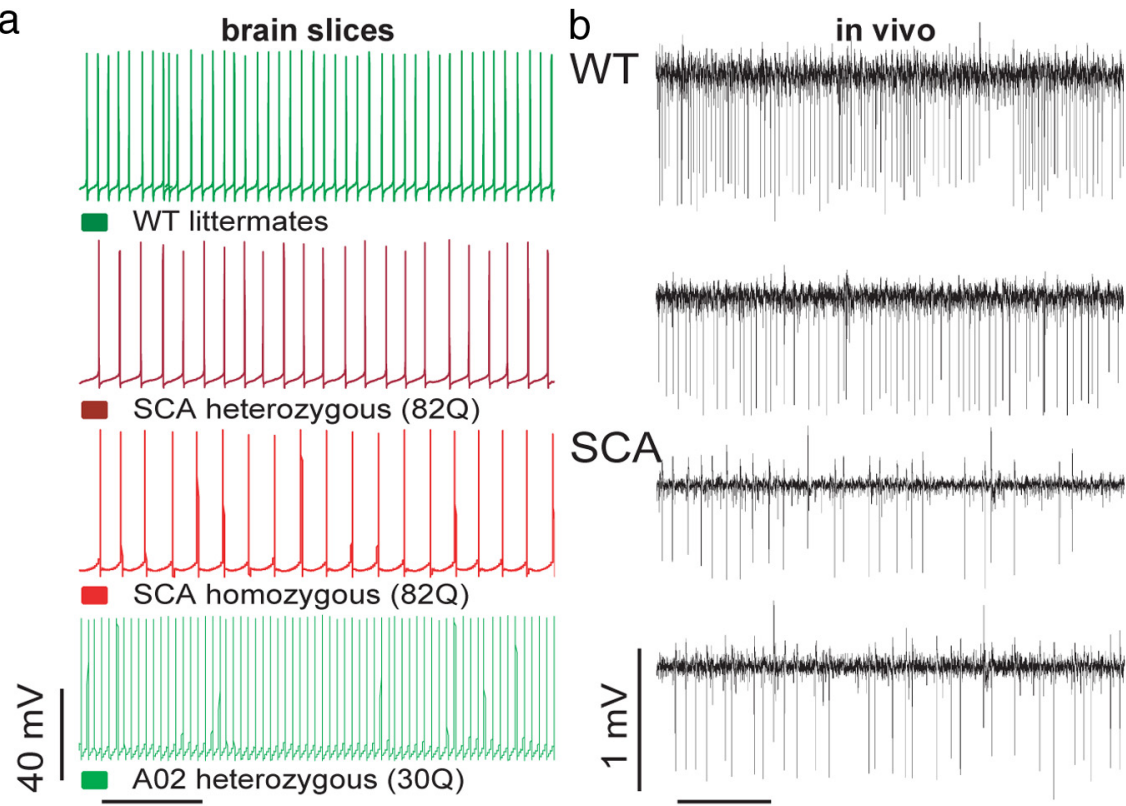

$\overline{0.2 \mathrm{~s}}$

C brain slices

d
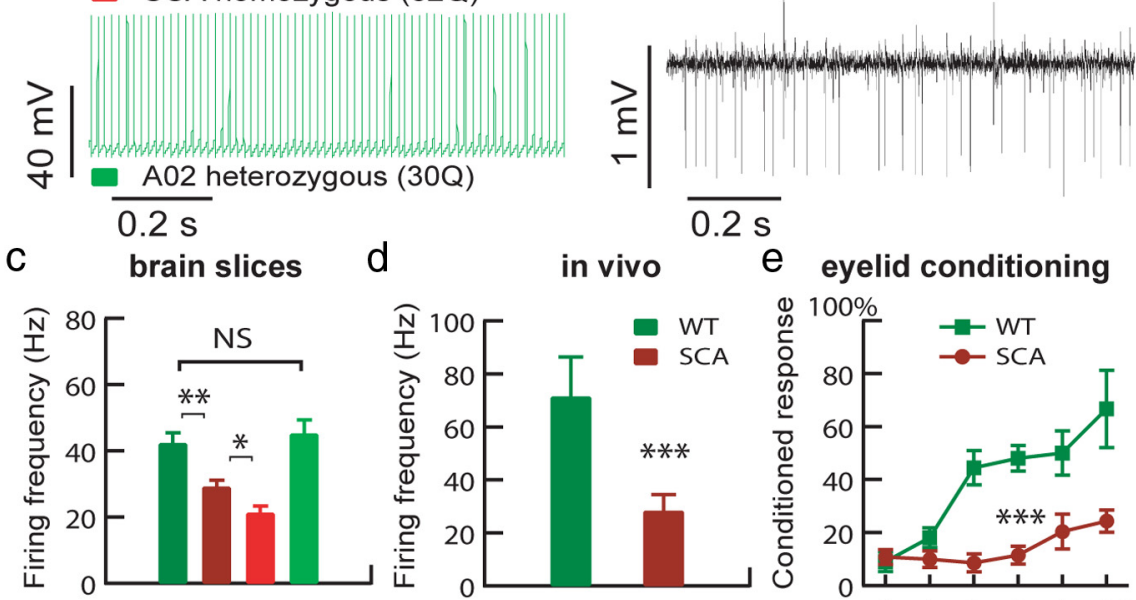

f

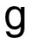

g
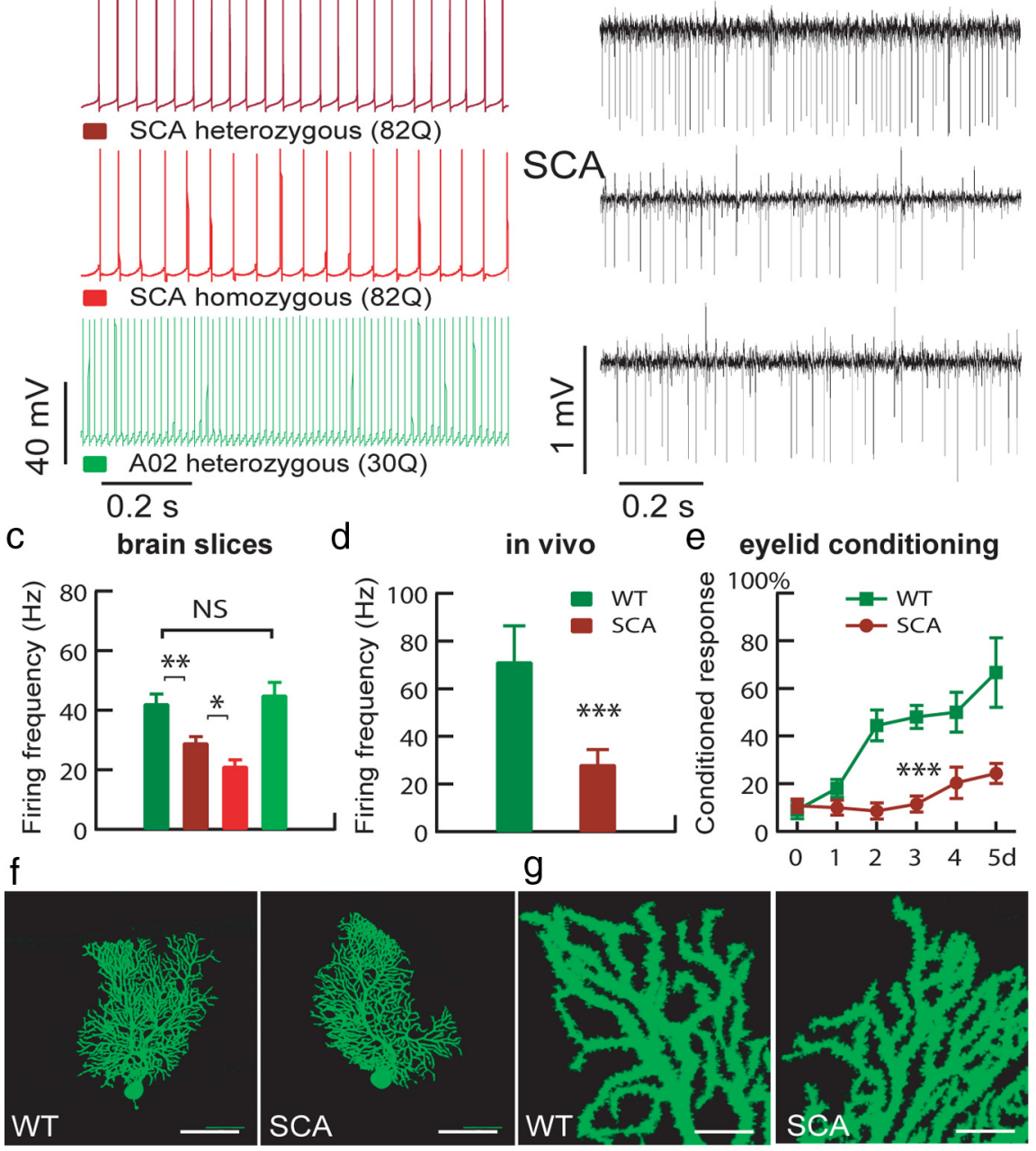

SCA

$0.2 \mathrm{~s}$

e eyelid conditioning

Figure 2. The firing frequency of $\mathrm{PCS}$ is reduced in presymptomatic SCA1 mice. $a$, Firing pattern of $\mathrm{PC}$ in brain slices from control mice (WT), mice heterozygous for human mutated ataxin1 [SCA heterozygous (820)], homozygous for human mutated ataxin1 [SCA homozygous (820)], and heterozygous for normal human ataxin1 [A02 heterozygous (30Q)]. $\boldsymbol{b}$, In vivo firing pattern of PCS from WT and SCA1 mice (heterozygous for human mutated ataxin1). c, Firing frequency of PCs in brain slices from WT mice ( $n=$ 18P(s), mice heterozygous for human mutated ataxin1 ( $n=35)$, homozygous for human mutated ataxin1 $(n=28)$, and heterozygous for normal human ataxin $1(n=24)$ (one-way ANOVA followed by Fisher's test, WT vs SCAhet, $p=0.025$; WT vs SCAhom, $p=0.00001$; WT vs A02, $p=0.55$; SCAhet vs SCAhom, $p=0.034 ;{ }^{*} p<0.05 ;{ }^{* *} p<0.01 ; * * * p<0.001$ ) (color code as in $\boldsymbol{a})$. $\boldsymbol{d}$, In vivo simple spikes frequency from WT $(n=35)$ and SCA1 $(n=35)$ PCs ( $t$ test, $p=0.00027)$. $\boldsymbol{e}$, Delayed acquisition of eyeblink conditioning in presymptomatic SCA1 mice. Eyeblink conditioning is assessed by the percentage of conditioned response (eyeblink after a sound alone) after days of conditioning sessions associating a sound (conditioned stimulus) to an air puff directed to the eye and causing an eyeblink (unconditioned stimulus) [WT mice ( $n=5)$ vs SCA1 ( $n=7)$; one-way ANOVA followed by Fisher's test, $p=0.00032$ ]. Error bars indicate SEM. $\boldsymbol{f}, \boldsymbol{g}$, The morphology of the Purkinje cell is not altered in presymptomatic SCA1 mice. $f$, Typical appearance of biocytin-loaded Purkinje cells from 3-week-old wild-type (left) and SCA1 (right) mice. Scale bar, $50 \mu \mathrm{m} . \boldsymbol{g}$, Typical appearance of biocytin-loaded dendrites from Purkinje cells from 3-week-old wild-type (left) and SCA1

Statistical analyses. Results are reported and illustrated as mean \pm SEM. The normal distribution of the data was checked using ShapiroWilk test. For comparisons of one variable between two groups, means and variance were compared using $t$ and $F$ tests, respectively. Comparison of one variable between more than two groups was made using one-way ANOVA. Analysis of rotarod motor performance and acquisition of eyeblink conditioning were made using two-way ANOVA. ANOVAs 
Table 2. In vivo recordings

\begin{tabular}{|c|c|c|c|c|c|c|c|}
\hline \multirow[b]{2}{*}{ Parameters } & \multirow[b]{2}{*}{ Unit } & \multicolumn{2}{|c|}{ WT (no. of cells $=35$ ) } & \multicolumn{2}{|c|}{ SCA1 (no. of cells $=35)$} & \multirow[b]{2}{*}{$t$ test } & \\
\hline & & Mean & SEM & Mean & SEM & & \\
\hline Simple spike frequency & $\mathrm{Hz}$ & 71.29 & 5.70 & 28.24 & 2.38 & $1.15 \mathrm{E}-08$ & $* * *$ \\
\hline CS frequency & $\mathrm{Hz}$ & 1.47 & 0.08 & 1.25 & 0.08 & 0.06 & NS \\
\hline CS duration & $\mathrm{ms}$ & 9.17 & 0.27 & 11.38 & 0.42 & 0.0001 & $* * *$ \\
\hline Pause duration after CS & $\mathrm{ms}$ & 12.94 & 0.57 & 27.42 & 1.81 & $6.42 \mathrm{E}-09$ & $* * *$ \\
\hline Simple spike rythmicity index & & 0.06 & 0.01 & 0.02 & 0.00 & 0.004 & $* *$ \\
\hline
\end{tabular}

In vivo recordings parameters of Purkinje cells from 4 - to 5 -week-old WT and SCA1 mice. CS, Complex spike.

NS, No significant difference $(p>0.05) ;{ }^{* *} p<0.01 ;{ }^{* * *} p<0.001$

were followed by least significant difference (LSD) Fisher's post hoc test in case of multiple comparisons. Proportions of cells between two groups were compared using Fisher's test. Comparison of $\mathrm{IK}_{\mathrm{A}}$ amplitude from the same cells before and after application of drugs or toxins was made using paired $t$ test. Statistical analysis of mEPSCs amplitude distribution was performed using the Kolmogorov-Smirnov two-sample test (Mini Analysis). Differences were considered statistically significant for values of $p<0.05\left({ }^{*} p<0.05 ;{ }^{* *} p<0.01 ;{ }^{* * *} p<0.001\right)$.

\section{Results}

Motor impairment precedes morphological changes and loss of PCs in SCA1 mice

We first established the chronological succession of neurological deficits, atrophy, and loss of PCs in SCA1 mice (Fig. 1). Threeweek-old control and SCA1 mice have similar motor behavior (Fig. 1a), cerebellar morphology (Fig. 1b), and Purkinje cell count $[100 \pm 6.15$ and $97.16 \pm 10.67 \%$ of wild-type count for control $(n=5)$ and SCA1 $(n=6)$ mice, respectively; $p=0.71]$. Five-week-old SCA1 mice display impaired motor performance (Fig. 1c) with normal cerebellar morphology (Fig. 1d) and cell count $[100 \pm 6.67$ and $94.06 \pm 5.84 \%$ of wild-type count for control $(n=5)$ and SCA1 $(n=5)$ mice, respectively; $p=0.52]$. Ten-week-old SCA1 mice display impaired motor behavior (Fig. 1e) and PC alterations such as ectopic and atrophic PCs and molecular layer shrinking (Fig. $1 \mathrm{f}$ ), but PC count is unchanged $[100 \pm 7.88$ and $85.14 \pm 7.77 \%$ of wild-type count for control $(n=5)$ and SCA1 $(n=5)$ mice, respectively; $p=0.21]$. Eighteenweek-old SCA1 mice display impaired motor behavior (Fig. $1 g$ ), cerebellar morphological alterations (Fig. 1h), and significant PC death $[100 \pm 7.21$ and $75.51 \pm 4.58 \%$ of wild-type count for $\operatorname{control}(n=11)$ and SCA1 $(n=9)$ mice, respectively; $p=0.014]$. In the following experiments, we will call 2- to 4 -week-old mice presymptomatic, 5-week-old mice early symptomatic, and 18week-old mice "old" or "late stage."

\section{The firing frequency of PCs is reduced in presymptomatic SCA1 mice}

We first studied the firing pattern of PCs at the presymptomatic stage. PCs exhibit a spontaneous electrical activity, characterized in slices by regular action potentials and consisting in vivo of simple spikes interrupted by a few complex spikes. PC firing rate is reduced in presymptomatic SCA1 mice both in slices and in vivo (Fig. 2; Tables 1,2). The expression of normal human ataxin-1 did not affect the firing frequency of PCs (Fig. 2a,c). The reduction in firing frequency was correlated with the amount of expressed mutated ataxin-1 as PCs from homozygous SCA1 mice were slower than PCs from heterozygous mice (Fig. 2a,c). In vivo, the reduced firing frequency of PCs in presymptomatic SCA1 mice (Fig. $2 b, d$ ) was associated with reduced rhythmicity index, longer complex spikes, and longer pauses following complex spikes (Table 2). As this reduction in PC firing frequency oc- curred in the absence of motor behavior impairment, we studied the functioning of the cerebellar circuitry by testing the acquisition of eyelid conditioning, a process that requires the precise integration of various stimuli by PCs (Green and Steinmetz, 2005). Presymptomatic SCA1 mice poorly learned the conditioned eyeblink response (Fig. 2e), suggesting that reduced firing frequency of PCs (or another dysfunction of these cells at this stage) is enough to impair the functioning of the whole cerebellar circuitry. PC volume [40.56 \pm 4.26 and $42.11 \pm 3.01 \mu \mathrm{m}^{3}$ in WT $(n=21)$ and SCA1 $(n=35)$ mice, respectively; $p=0.85]$ and dendritic spine density $(1.42 \pm 0.03$ and $1.38 \pm 0.05$ spines $/ \mu \mathrm{m}$ in WT and SCA1 mice, respectively; two dendrites were analyzed per cell; $p=0.45$ ) were normal in presymptomatic SCA1 mice (Fig. $2 f, g$ ), ruling out subtle morphological changes as a cause for altered neuronal excitability.

\section{The efficiency of the glutamatergic parallel fiber to PC synapse is reduced in presymptomatic SCA1 mice}

As mutated ataxin-1 alters the expression of genes involved in glutamate signaling in presymptomatic SCA1 mice (Lin et al., 2000; Serra et al., 2004), we tested whether glutamatergic transmission was functionally impaired in presymptomatic SCA1 mice. Analysis of the relationship between increasing stimulation of parallel fibers and the initial slope of evoked EPSCs (Servais et al., 2007) indicated that the strength of the parallel fiber-PC synapse was decreased in presymptomatic SCA1 mice (Fig. $3 a, b$ ), with mean slope coefficient values in PCs of $2.88 \pm 0.43$ and $1.53 \pm 0.38 \mathrm{mHz}$ for WT $(n=10)$ and presymptomatic SCA1 $(n=13)$ mice, respectively $(p=0.029)$. Paired-pulse facilitation, a presynaptic event, was identical in control and SCA1 mice (Fig. $3 c, d)$. These results were confirmed by recordings of miniature currents (mEPSCs) caused by the spontaneous and probabilistic release of neurotransmitter vesicles from presynaptic neurons (Fig. 3e,f). Cumulative mean amplitude distributions showed a shift toward smaller amplitudes of mEPSC recorded from SCA1 PCs (Fig. 3f), while mEPSC frequencies were similar in WT and SCA1 groups (Fig. $3 g$ ) $[0.74 \pm 0.05$ and $0.66 \pm 0.08 \mathrm{~Hz}$ for WT $(n=6)$ and SCA1 $(n=7)$ mice, respectively; $p>0.05]$. mEPSCs recorded from SCA1 PCs also showed slower kinetics (time to rise and to decay) (Fig. 3h,i). These data suggest that the reduction in the strength of the parallel fiber-PC synapse is caused by an alteration of the postsynaptic side of the synapse.

\section{The amplitude of the A-type potassium current $\left(\mathrm{IK}_{\mathrm{A}}\right)$ is increased in PCs from presymptomatic SCA1 mice}

To further study the mechanisms of reduction in $\mathrm{PC}$ firing rate in presymptomatic SCA1 mice, spontaneous and depolarizationinduced electrical activities were analyzed. Action potential parameters were similar in WT and SCA1 mice, suggesting that currents involved in the shaping of action potentials are not al- 
tered (Table 1). During excitability recordings, all PCs from WT mice and most PCs from SCA1 mice showed a smooth progressive depolarization preceding action potentials, but a significant proportion of SCA1 cells showed an irregular plateau potential delaying the first action potential (Fig. 4a) during the first depolarizing steps evoking a train of action potentials from a holding potential of -80 $\mathrm{mV}$. This plateau was not observed in control cells ( 7 of 43 SCA1 cells vs 0 of 25 cells; $p=0.041$, Fisher's test). A similar plateau potential was previously described in most PCs from 3- to 6-monthold SCA1 mice (Inoue et al., 2001) and is suggestive of fast activation and inactivation of an A-type potassium conductance $\left(\mathrm{IK}_{\mathrm{A}}\right)$ (Hounsgaard and Midtgaard, 1988; Klee et al., 1995). Accordingly, when recorded, this plateau current was reversibly abolished by low doses of 4aminopyridine and could be recruited by a hyperpolarizing prepulse (Fig. $4 b$ ). Interestingly, the proportion of PCs displaying this irregular plateau potential is higher in older SCA1 mice, as if its occurrence reflected the progression of the disease and was correlated with disease severity: $16 \%$ of PCs display the plateau in presymptomatic mice, $45 \%$ in early symptomatic SCA1 mice ( 5 of 11 vs 0 of 10 in controls; $p=0.035$, Fisher's test), and $>80 \%$ in 3 - to 6-month-old SCA1 mice (Inoue et al., 2001). IK $\mathrm{A}$ peak amplitude was isolated in PCs from presymptomatic SCA1 mice by the subtraction of potassium currents evoked by a depolarization to $-20 \mathrm{mV}$ after hyperpolarized $(-80$ $\mathrm{mV})$ and depolarized $(-40 \mathrm{mV})$ conditioning steps (200 ms) from a holding potential of $-70 \mathrm{mV}$. IK $\mathrm{A}$ peak current was significantly increased in PCs from SCA1 mice (Fig. 4c) with amplitude of $525.1 \pm$ 35.2 and $687.5 \pm 27.7 \mathrm{pA}$ for WT $(n=16)$ and SCA1 $(n=18)$ mice, respectively $(p=0.00088)$. Toxins were used to determine which of the Kv1 and Kv4 channels (Serôdio and Rudy, 1998; Sacco and Tempia, 2002; Wang and Schreurs, 2006) mediate $\mathrm{IK}_{\mathrm{A}}$ in PCs (Fig. $4 d$ ). Inhibition of all $\mathrm{Kv} 4$ channels by application of phrixotoxin reduced $\mathrm{IK}_{\mathrm{A}}$ by $\sim 90 \%$, while the specific inhibition of Kv4.2 channels by stromatoxin had no effect. Inhibition of the main members of the Kv1 family reduced $\mathrm{IK}_{\mathrm{A}}$ by $\sim 10 \%$ (hongotoxin inhibiting Kv1.1, 1.2, 1.3, and 1.6). The toxins had similar effects when applied on PCs from control and SCA1 mice. $\mathrm{IK}_{\mathrm{A}}$ is thus mainly mediated by Kv4.1 and/or Kv4.3 channels in PCs from both control and SCA1 mice. The expression of Kv4.1 (Fig. $5 a-c$ ), Kv4.2 (Fig. $5 d-f$ ), and Kv4.3 (Fig. $5 g-i$ ) was studied in control and presymptomatic SCA1 mice by immunohistochemistry, Western blotting, in situ hybridization, and qRT-PCR per-

a

C

e
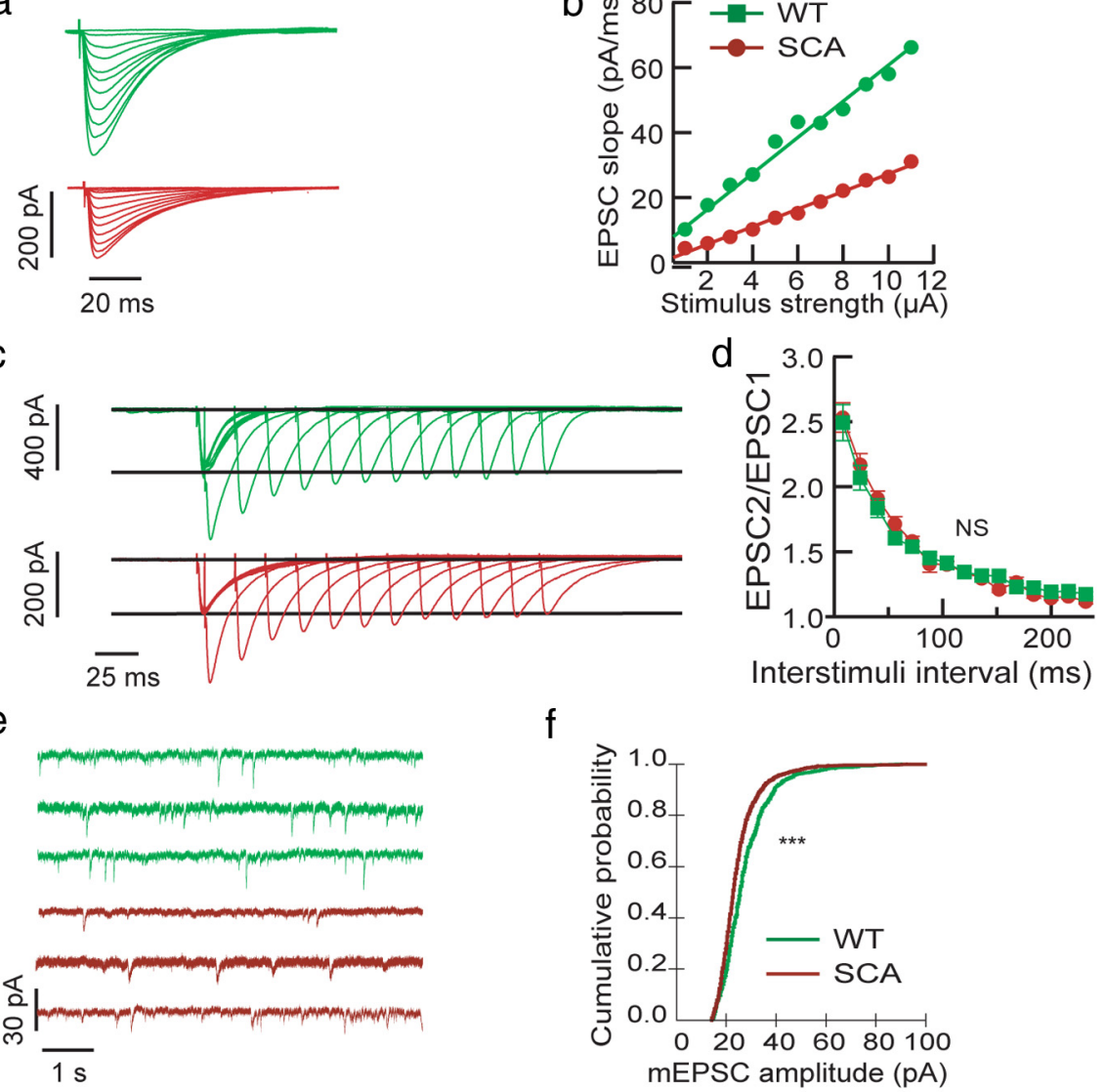

f

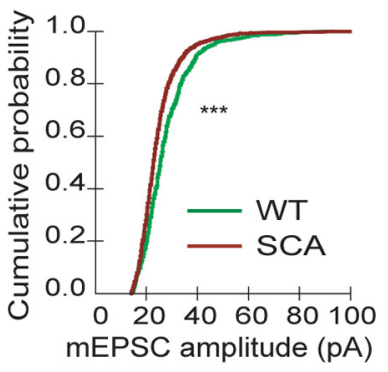

g

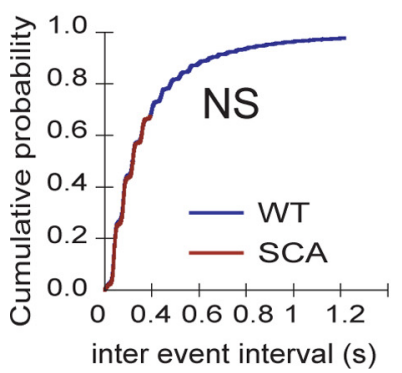

$\mathrm{h}$

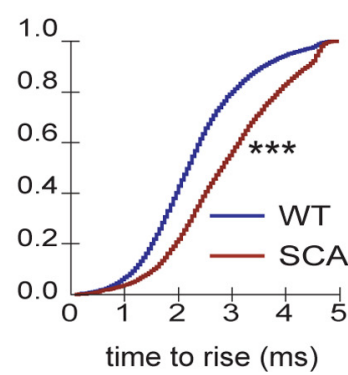

i
Figure 3. Decreased synaptic strength of the parallel fiber-PC synapse in presymptomatic SCA1 mice. $\boldsymbol{a}$, Typical input- output relationships obtained from $P C s$ in response to an increasing stimulation of parallel fibers in a control (top panel) and a SCA1 (bottom panel) PC. Negative current pulses ranging from 0 to $11 \mu \mathrm{A}$ were delivered in ascending order. $\boldsymbol{b}$, Linear relationship between evoked EPSC initial slope and stimulus intensity in the cells illustrated in $\boldsymbol{a}$. $\boldsymbol{c}$, Typical traces of paired-pulse facilitation, a short-term synaptic plasticity reflecting the probability of glutamate release from the presynaptic parallel fibers after a second stimulation that closely follows a first one, in control and SCA1 mice. $\boldsymbol{d}$, The paired-pulse facilitation ratio was expressed as the maximal amplitude of the second EPSC divided by the maximal amplitude of the first EPSC (WT, $n=10$; SCA, $n=12$; one-way ANOVA followed by Fisher's test, $p=0.9$ ). This result suggests that the presynaptic side of the parallel fiber-Purkinje cell synapse is not altered. Error bars indicate SEM. $\boldsymbol{e}$, Representative mEPSCs from three different WT and SCA PCs. $\boldsymbol{f}$, Cumulative mean amplitude distributions of mEPSCs in PCs from WT $(n=6)$ and SCA1 $(n=7)$ mice showing a significant shift (KolmogorovSmirnov test, ${ }^{* * *} p<0.001$ ) toward smaller amplitudes in SCA1 mice. $g$, mEPSC frequency is not different in PCs from WT and SCA 1 mice. $\boldsymbol{h}, \boldsymbol{i}, \mathrm{mEPSCs}$ from SCA1 mice show slower kinetics (longer time to rise and time to decay) compared with WT mice (Kolmogorov-Smirnov test, $\left.{ }^{* * *} p<0.001\right)$.

formed both on microdissected PCs and on whole cerebella. As previously described (Serôdio and Rudy, 1998), PCs express high levels of Kv4.3, very low levels of Kv4.1, and no Kv4.2 channels (in agreement with the absence of effect of stromatoxin on the amplitude of $\left(\mathrm{K}_{\mathrm{A}}\right)$. No increase in the expression of $\mathrm{Kv} 4$ channels was found in SCA1 mice (Fig. 5) [quantitative RT-PCR analysis in microdissected Purkinje cells of expression of Kv4.1, $0.80 \pm 0.46$ and $0.55 \pm 0.32$ for WT $(n=3)$ and SCA1 $(n=3)$ mice, respec- 
a
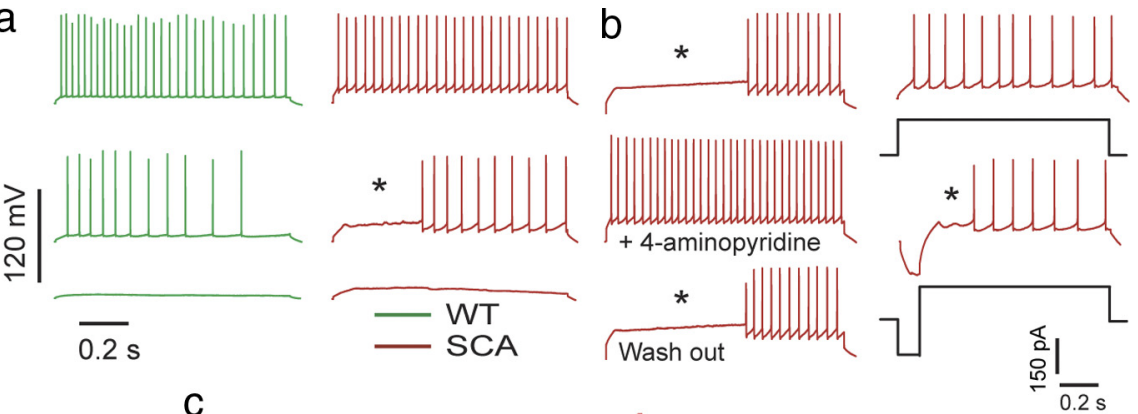

C

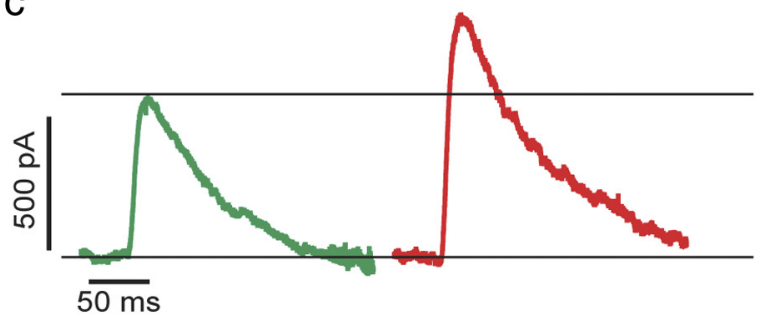

d

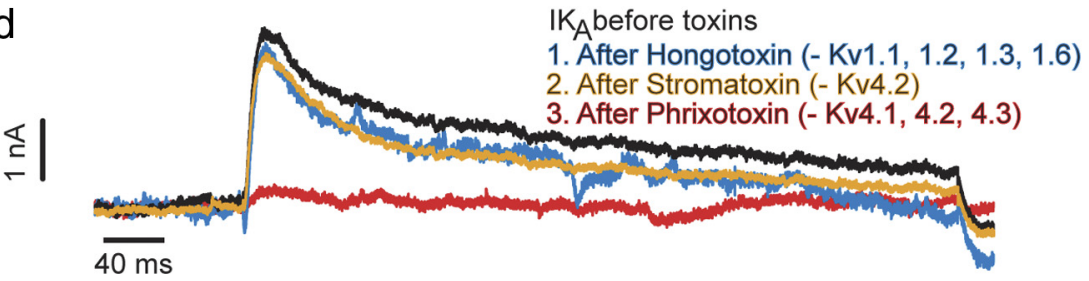

Figure 4. Increased II $\mathrm{K}_{\mathrm{A}}$ in PCs from presymptomatic SCA1 mice. $\boldsymbol{a}$, Typical PC excitability recordings (50, 100, 150 pA current injections from a holding potential of $-80 \mathrm{mV}$ ) from a wild-type (left panel) and a SCA1 (right panel) mouse. A significant proportion of SCA1 cells displayed an irregular plateau potential delaying the first action potential. The asterisk $\left(^{*}\right)$ indicates the irregular plateau potential in $\boldsymbol{a}$ and $\boldsymbol{b}$. $\boldsymbol{b}$, The irregular plateau potential is reversibly abolished by $50 \mu \mathrm{m}$ 4-aminopyridine (left panel) and can be elicited by a hyperpolarizing prepulse (right panel). $c$, Typical current trace of $I K_{A}$ evoked in a PC from a WT or $\mathrm{SCA} 1$ mouse by a $60 \mathrm{mV}$ depolarization from a $-80 \mathrm{mV}$ holding potential after subtraction of sustained inactivating $\mathrm{K}^{+}$currents $\boldsymbol{d}$, Typical IK $\mathrm{K}_{\mathrm{A}}$ current traces from a SCA1 PC before and after successive application of hongotoxin, stromatoxin, and phrixotoxin.

\section{Surface Kv4.3 channels are more} abundant in PCs from presymptomatic SCA1 mice

As the reduction in $\mathrm{IK}_{\mathrm{A}}$ amplitude by glutamate and AMPA could be caused either by an inhibitory regulation or by internalization of Kv4.3 channels, we determined total and surface levels of Kv4.3 in the anterior vermis. Kv4.3 expression in the anterior vermis reflects expression within PCs as granule cells do not express Kv4.3 in this part of the cerebellum, while they do in lateral and posterior parts of the cerebellum (Fig. 5h) (Serôdio and Rudy, 1998). PCs from presymptomatic SCA1 mice show a moderate increase (1.5-2 times) in total Kv4.3 and a $>10$ times increase in surface Kv4.3 (Fig. 6j). Level of Kv4.3 mRNA normalized to the wild-type value is normal in the anterior vermis of presymptomatic SCA1 mice [1.07 \pm 0.09 and $0.94 \pm 0.09$ for WT $(n=6)$ and SCA1 $(n=6)$ mice, respectively; $p=0.31$ ], suggesting that an accumulation of $\mathrm{Kv} 4.3$ channels is associated with the increase in surface expression. Altered glutamate receptors stimulation might lead to a reduction in the internalization of $\mathrm{Kv} 4.3$ channels in SCA1.

Acute treatment with aminopyridines normalizes the firing frequency of PCs and the motor phenotype of early symptomatic but not old SCA1 mice We then tested whether neuronal electrical dysfunctions could contribute to the

tively; Kv4.2, $1.21 \pm 0.70$ and $1.19 \pm 0.69$ for WT $(n=3)$ and SCA1 $(n=3)$ mice, respectively; and Kv4.3, $1.11 \pm 0.64$ and $1.25 \pm 0.72$ for WT $(n=3)$ and SCA1 $(n=3)$ mice, respectively; quantitative RT-PCR analysis in whole cerebella of Kv4.1, $0.74 \pm$ 0.09 and $0.70 \pm 0.09$ for WT $(n=5)$ and SCA1 $(n=5)$ mice, respectively; Kv4.2, $0.77 \pm 0.08$ and $0.76 \pm 0.05$ for WT $(n=5)$ and SCA1 $(n=5)$ mice, respectively; and Kv4.3, $0.83 \pm 0.08$ and $0.82 \pm 0.08$ for WT $(n=5)$ and SCA1 $(n=5)$ mice, respectively; $p>0.05$ for all]. These results reasonably ruled out Kv 4 channels overexpression as the cause for the increased $\mathrm{IK}_{\mathrm{A}}$ observed in SCA1 mice.

\section{Glutamate and AMPA normalize $\mathrm{IK}_{\mathrm{A}}$ amplitude in PCs from presymptomatic SCA1 mice}

As Kv4 channels do not appear overexpressed in SCA1, the observed increase in $\mathrm{IK}_{\mathrm{A}}$ might be explained by impaired trafficking of Kv4.3 channels. As glutamate receptor stimulation induces the internalization of $\mathrm{Kv} 4$ channels in hippocampal neurons (Kim et al., 2007; Lei et al., 2008), we next tested whether glutamate could affect $\mathrm{IK}_{\mathrm{A}}$ amplitude in cerebellar PCs. Glutamate and AMPA, but not NMDA (in the presence of $1.19 \mathrm{~mm} \mathrm{MgSO}_{4}$ in the extracellular solution), reduced $\mathrm{IK}_{\mathrm{A}}$ by $\sim 30 \%$ in WT PCs and $\sim 45 \%$ in SCA1 PCs (Fig. 6a-f). Interestingly, $\mathrm{IK}_{\mathrm{A}}$ amplitude was no longer significantly increased in SCA1 PCs after application of saturating concentration of glutamate and AMPA (Fig. $6 a-d$ ). Glutamate receptor stimulation did not affect sustained potassium current (Fig. $6 g-i$ ). altered motor impairment in early symptomatic SCA1 mice. Treatment of 5-week-old SCA1 mice with DiAP, a potassium channel blocker with high affinity for $\mathrm{IK}_{\mathrm{A}}$, normalized the firing frequency of SCA1 PCs and the motor performance of the animals (Fig. $7 a-c$ ). No significant effect was found in WT mice. AP, another $\mathrm{IK}_{\mathrm{A}}$ blocker, also improved the motor behavior of early symptomatic SCA1 mice (Fig. $7 d$ ). Treatment of 18 -week-old SCA1 mice with DiAP also normalized the firing frequency of SCA1 PCs; however, it did not improve the motor performance (Fig. $7 f-j$ ), suggesting that impairment of motor coordination in old SCA1 mice is no longer primarily caused by electrophysiological dysfunction, but by later events such as PC atrophy and death. To precise how aminopyridines are able to reincrease the firing rate of PCs, we applied 4-aminopyridines onto cerebellar slices from 4- to 5-week-old WT mice while recording mEPSCs from PCs. We did not observe any significant effect of the application of 4-aminopyridine on the amplitude or frequency of mEPSCs, suggesting that aminopyridines do not act through a potentiation of glutamatergic synapses onto PCs (Fig. 8).

\section{Chronic treatment with DiAP improves motor phenotype in old SCA1 mice}

To test whether early neuronal dysfunction contributes to late neurodegenerative events such as morphological alterations, SCA1 mice were chronically treated with DiAP from presymptomatic until advanced disease stage (4-18 weeks). Chronically treated old SCA1 mice displayed improved motor 

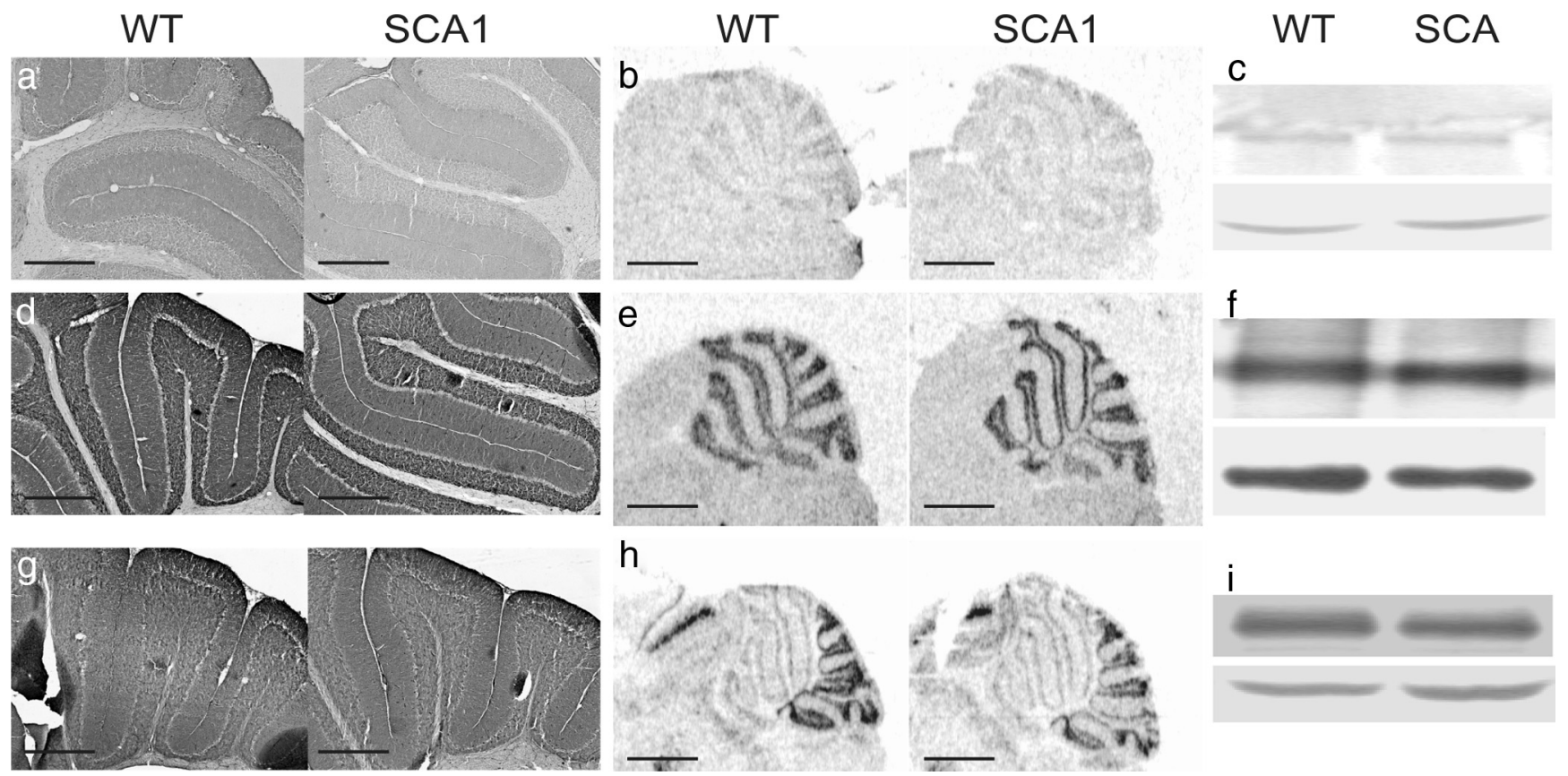

Figure 5. Kv4 channels are not overexpressed in presymptomatic SCA1 mice. $\boldsymbol{a}, \boldsymbol{d}, \boldsymbol{g}$, Immunohistochemistry using antibody directed against Kv4.1 (a), Kv4.2 (d), or Kv4.3 (g) in 5-week-old WT and SCA1 mice. Scale bar, $250 \mu \mathrm{m} . \boldsymbol{b}, \boldsymbol{e}, \boldsymbol{h}$, In situ hybridization using probes directed against Kv4.1 (b), Kv4.2 (e), or Kv4.3 (h) in 5-week-old WT and SCA1 mice. Scale bar, 1 mm. $\boldsymbol{c}, \boldsymbol{f}, \boldsymbol{i}$, Western blotting of whole cerebellum using antibody directed against Kv4.1 (c), Kv4.2 (f), or Kv4.3 (i) in 5-week-old WT and SCA1 mice. Actin was used to normalize protein loading (bottom band).

performance (Fig. $7 k-m$ ) and increased PC firing frequency (Fig. $7 n, o$ ). Chronic administration was as effective as single injection to improve motor performance in 5-week-old SCA1 mice (Fig. 7e).

\section{Chronic treatment with DiAP preserves the thickness of the molecular layer, the volume, and the dendritic spine density of PCs, and increases cerebellar levels of BDNF}

So far, the data suggest that increasing the firing frequency of PCs from a presymptomatic stage improves the motor behavior of old SCA1 mice. As behavior is no longer determined by electrophysiological dysfunction but likely by later events such as PC atrophy in old SCA1 mice, we assessed whether chronic treatment with DiAP protected SCA1 mice from PC atrophy. Chronic treatment with 3,4-DiAP improved the thickness of the molecular layer, the volume, and the density of dendritic spines in PCs from old SCA1 mice (Fig. 9a-c). Recordings of the parallel fiber-PC synapse showed that it was better preserved in chronically treated SCA1 mice (Fig. 9d), indicating a functional correlate to morphological improvement. Neuronal activity influences dendritic remodeling through neurotrophic factors like BDNF (McAllister et al., 1996; Balkowiec and Katz, 2002). BDNF is abundantly expressed in the cerebellum and in PCs (Dieni and Rees, 2002; Ohira et al., 2004), is produced in an activity-dependent mode (Balkowiec and Katz, 2002), and influences both the dendritic morphology of PCs (Adcock et al., 2004) and the parallel fiber-PC synapse (Carter et al., 2002; Furutani et al., 2006; Hisatsune et al., 2006). BDNF cerebellar levels were decreased in old SCA1 mice compared with control mice, and normalized in the cerebellum of chronically treated SCA1 mice (Fig. 9e). By restoring a normal PC neuronal activity, aminopyridines may in turn increase the cerebellar production of BDNF that may consequently protect PCs against dendritic atrophy. Interestingly, chronic treatment with DiAP did not reduce PC death in old SCA1 mice, suggesting that pathogenic mechanisms leading to cell atrophy and to cell death may be distinct (Fig. 9f). Finally, using an antibody recognizing expanded polyglutamine tract (1C2), we determined the protein level of ataxin-1 in WT or SCA1 mice chronically treated with saline or DiAP and did not observe any effect of the chronic treatment on the protein level of expanded ataxin-1 (Fig. $9 g, h$ ).

\section{Discussion}

This study suggests that early neuronal dysfunction may play a pathogenic role in neurodegenerative disorders, including those that are not primarily caused by ion channel mutations. In the studied mouse model of SCA1, restoring a normal neuronal firing frequency from an early presymptomatic stage improved later neurological deficits and neuronal atrophy. Early neuronal dysfunction may constitute a relevant therapeutic target for some neurodegenerative disorders.

PCs from presymptomatic SCA1 mice show slower firing rate, reduced excitatory glutamatergic inputs, and higher A-type potassium current $\left(\mathrm{IK}_{\mathrm{A}}\right)$ that negatively controls firing frequency. We show that $\mathrm{IK}_{\mathrm{A}}$ is likely sustained by $\mathrm{Kv} 4.3$ channels in PCs, but we found no overexpression of $\mathrm{Kv} 4$ channels in SCA1 mice at the mRNA level. Impaired glutamate receptor stimulation could alter the trafficking of Kv4 channels and be responsible for the increased $\mathrm{IK}_{\mathrm{A}}$. Indeed, we show that glutamate and AMPA reduce $\mathrm{IK}_{\mathrm{A}}$ in PCs and that saturating concentrations of glutamate and AMPA normalize $\mathrm{IK}_{\mathrm{A}}$ amplitude in SCA1 mice. Furthermore, presymptomatic SCA mice show a major increase in surface Kv4.3 channels in the anterior vermis. In hippocampal neurons, the internalization of $\mathrm{Kv} 4.2$ channels after stimulation of glutamate receptors requires NMDA activation (Kim et al., 2007) and the presence in the channel sequence of a dileucine motif and a specific serine phosphorylated through signaling cascades that are not fully identified (Hammond et al., 2008). Since these motifs are evolutionary conserved in Kv4.3 (Rivera et al., 2003; van der Heyden et al., 2006), it is possible that, in PCs, AMPA activation results in voltage-dependent calcium channel activation and 


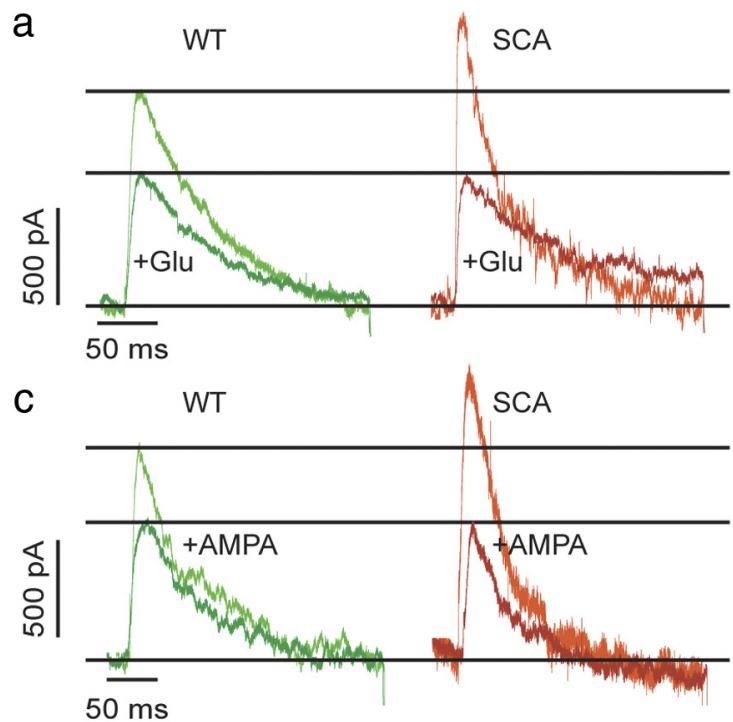

e
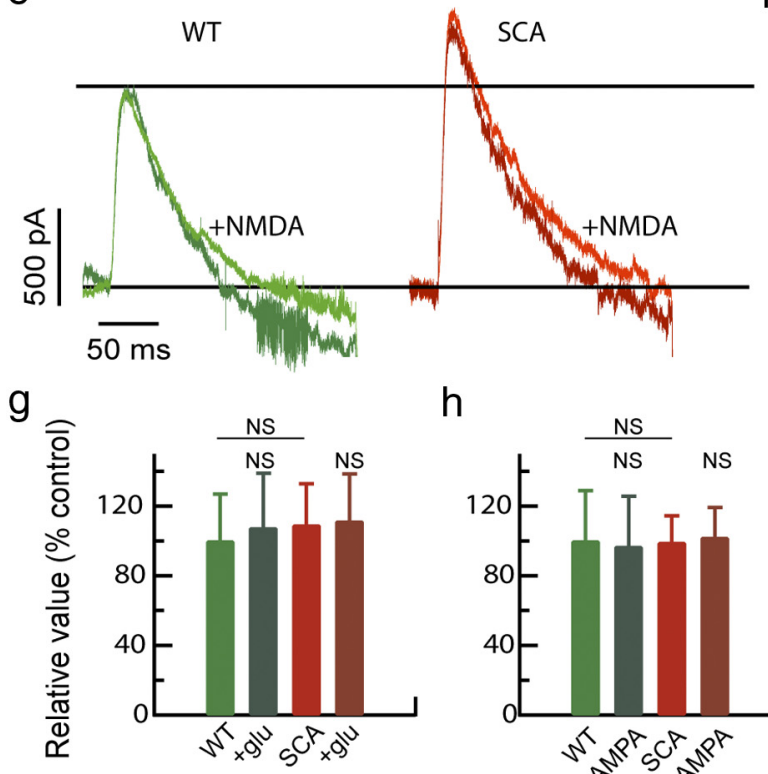

$\mathrm{h}$

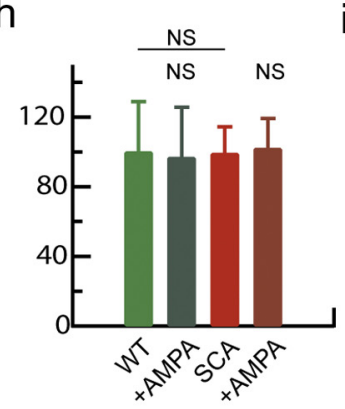

j

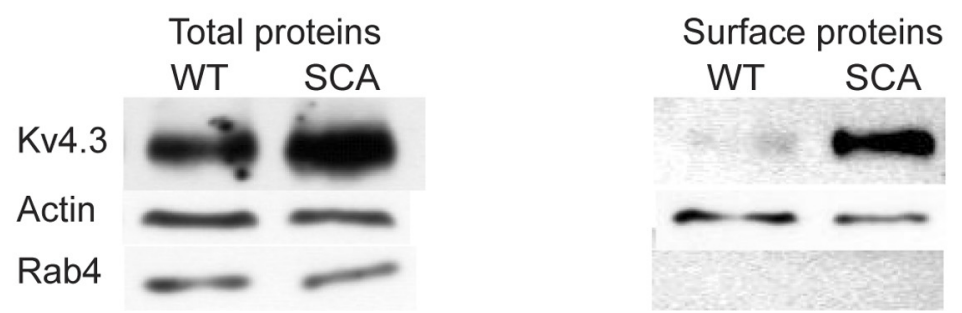

Figure 6. Altered glutamatergic transmission is associated with the increased I $K_{A}$ and more abundant surface Kv4.3 channels in PCs from presymptomatic SCA1 mice. $\boldsymbol{a}$, Typical IK $\mathrm{K}_{\mathrm{A}}$ current trace before and after application of glutamate $(50 \mu \mathrm{M})$ on a PC from a WT or SCA1 mouse. $\boldsymbol{b}$, Normalized $I \mathrm{~K}_{\mathrm{A}}$ amplitudes showing that glutamate significantly reduces peak $\mathrm{IK}_{\mathrm{A}}(5$ cells/group, paired $t$ test, WT pre vs post $p=0.009 ; S C A$ pre vs post, $p=0.0006)$ and suppresses the difference in $I_{A}$ between WT and SCA1 PCs ( $t$ test, WT vs SCA before glutamate, $p=0.036$; WT vs SCA after glutamate, $p=0.4)$. c, Typical IK $K_{A}$ current trace before and after application of AMPA (50 $\mu \mathrm{m})$ on a PC from a WT or SCA1 mouse. $\boldsymbol{d}$, Normalized IK $\mathrm{K}_{\mathrm{A}}$ amplitudes showing that AMPA significantly reduces peak $I K_{A}(5$ cells/group, paired $t$ test, WT pre vs post $p=0.002 ; S C A$ pre vs post, $p=0.001$ ) and suppresses the difference in IK $\mathrm{K}_{A}$ between WT and SCA1 PCs ( $t$ test, WT vs SCA before AMPA, $p=0.04$; WT vs SCA after AMPA, $p=0.9$ ). $e$, Typical IK $\mathrm{K}_{A}$ current trace before and after application of NMDA $(20 \mu \mathrm{M})$ on a PC from a WT or SCA1 mouse. $f$, Normalized IK amplitudes showing that NMDA has no effect on peak IK $\mathrm{A}_{A}(5$ cells/group, paired $t$ test, WT pre vs post, $p=0.4 ; \mathrm{SCA}$ pre vs post, $p=0.3$; WT pre vs SCA pre, $p=0.04) . \boldsymbol{g}-\boldsymbol{i}$, Normalized amplitudes of sustained $\mathrm{K}^{+}$current evoked by a depolarization to $-20 \mathrm{mV}$ from a holding potential of $-40 \mathrm{mV}$ showing the absence of effect of glutamate, AMPA, and NMDA and the absence of difference between WT and SCA1 PCS [5 cells/group, experiments with glutamate $(\boldsymbol{g}$ ), WT pre vs post, $p=0.33$ (paired $t$ test), SCA pre vs post, $p=0.73$, WT vs SCA, $p=$ 0.42 ( $t$ test); experiments with AMPA ( $\boldsymbol{h}$ ), WT pre vs post, $p=0.52$, SCA pre vs post, $p=0.51$, WT vs $S C A, p=0.91$; experiments increase in intracellular calcium levels without requirement for NMDA, leading to the initiation of intracellular cascades, phosphorylation, and internalization of Kv4.3 channels. As mutant ataxin- 1 alters the expression of genes involved in glutamate signaling and in AMPA receptors trafficking very early in SCA1 mice (Lin et al., 2000; Serra et al., 2004), this transcriptional effect of mutant ataxin-1 could constitute the primum movens leading to early neuronal dysfunction.

At the early symptomatic stage characterized by poor motor coordination without cell atrophy or death, aminopyridines normalize PC firing rate and motor behavior, suggesting that early neuronal electrical dysfunction causes neurological deficits at this stage. While acute treatment of old SCA1 mice also normalizes PC firing frequency, it fails to improve motor behavior, suggesting that deficits are likely supported by neuronal atrophy and loss at this late stage. Chronic treatment with DiAP from an early stage partially protects old SCA1 mice against motor impairment and partially preserves PC morphology, although cell death is not affected. The integrity of the parallel fiber-PC synapse also appears protected by a chronic treatment with DiAP.

The electrical activity of mature PCs improves their dendritic growth (Schilling et al., 1991) via neurotrophic factors like BDNF. BDNF is abundantly expressed in the cerebellar cortex (Dieni and Rees, 2002; Ohira et al., 2004), is produced in parallel with neuronal activity (Balkowiec and Katz, 2002), and influences the morphology of PCs and the parallel fiber-PC synapse (Carter et al., 2002; Adcock et al., 2004; Furutani et al., 2006; Hisatsune et al., 2006). Cerebellar BDNF level is reduced in the cerebellum of old SCA 1 mice and chronic treatment with DiAP restores BDNF levels, providing an explanation for the morphological protection afforded by aminopyridines. We did not determine whether BDNF is produced only by PCs or by both granule cells and PCs [by direct cross talk between these cells, or because granule cells sense an altered synaptic activity at the parallel fiber-PC synapse (Gatchel et al., 2008)] or whether other

$\leftarrow$

with NMDA (i), WT pre vs post, $p=0.47$, SCA pre vs post, $p=0.31$, WT vs $S C A, p=0.22]$. Error bars indicate SEM. $\boldsymbol{j}$, Total and surface levels of Kv4.3 channels were determined in the anterior vermis by Western blotting and biotinylation assay. Endogenous Rab4 is shown as cytoplasmic control; actin is shown as loading control. ${ }^{*} p<0.05 ;{ }^{* *} p<0.01$; ${ }^{* * *} p<0.001$. 

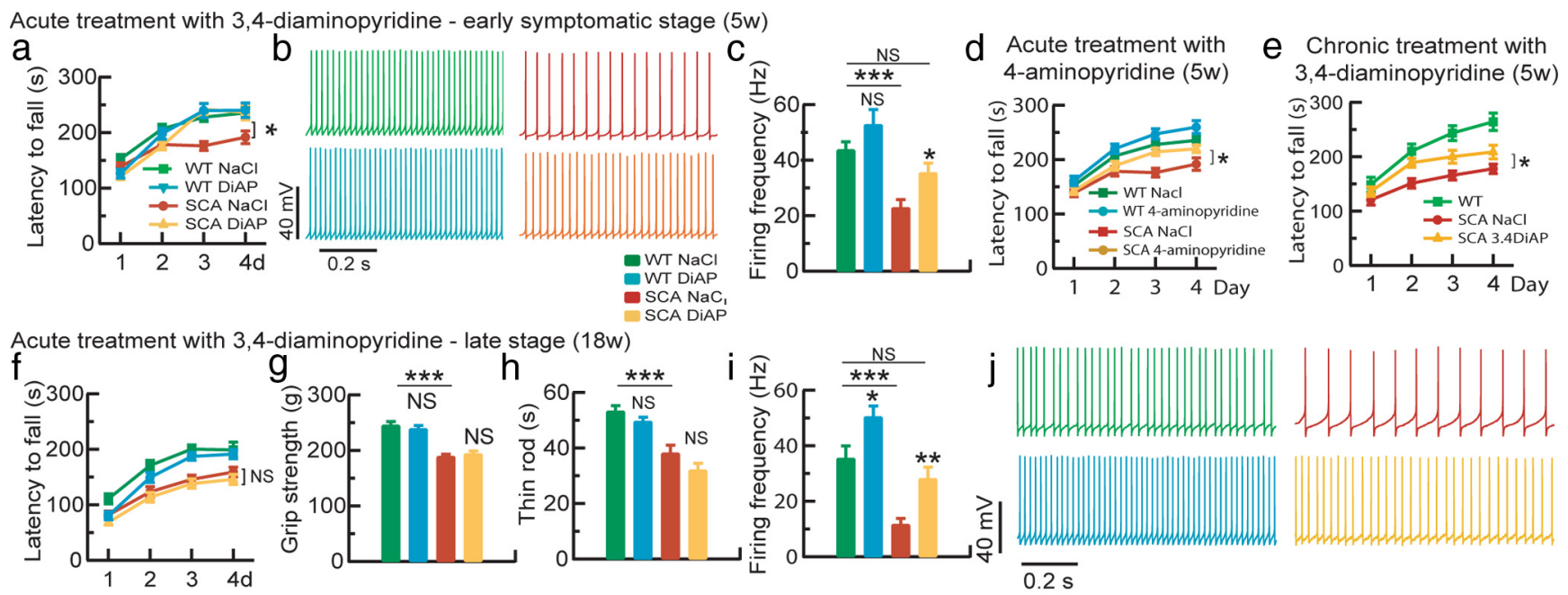

Chronic treatment with 3,4-diaminopyridine - late stage (18w)
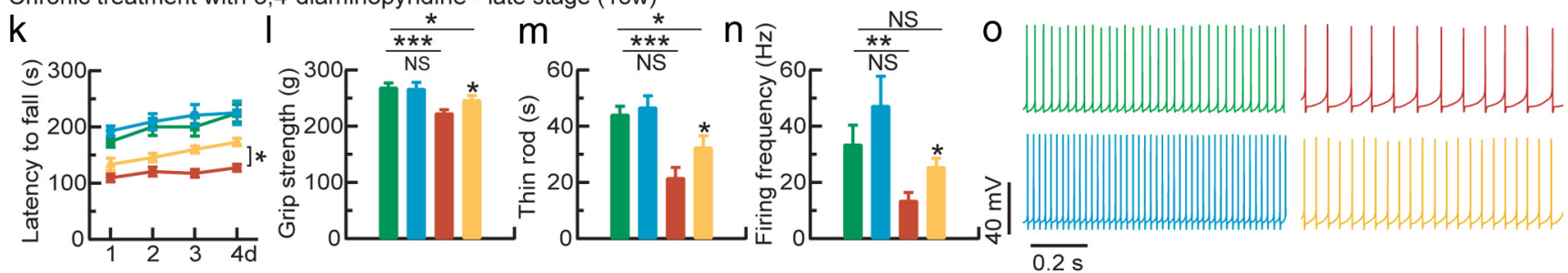

Figure 7. IK $\mathrm{K}_{\mathrm{A}}$ blockers normalize PCs firing frequency in SCA1 mice, normalize the motor phenotype of early symptomatic SCA1 mice, and improve the motor phenotype of old SCA1 mice only if administered chronically. $\boldsymbol{a}$, DiAP normalizes the latency to fall from an accelerated rotarod of 5-week-old SCA1 mice but has no significant effect on 5-week-old control mice (WT NaCl, $n=24$; WT DiAP, $n=23 ;$ SCA NaCl, $n=24 ;$ SCA DiAP, $n=23$; WTNaCl vs WTDiAP, $p=0.68$; WTNaCl vs SCANaCl, $p=0.0035 ;$ WTNaCl vs SCADiAP, $p=0.11 ;$ SCANaCl vs SCADiAP, $p=0.04) . \boldsymbol{b}$, Typical firing pattern of PCs in brain slices from 5-week-old control or SCA1 mice after injection of $\mathrm{NaCl}$ or DiAP. c, Firing frequency of PCs from 5-week-old wild-type or SCA1 mice subcutaneously injected with DiAP or saline (WT NaCl, $n=12 ;$ WT DiAP, $n=10 ;$ SCA NaCl, $n=11 ;$ SCA DiAP, $n=8 ;$ WT NaCl vs WT DiAP, $p=0.09 ;$ WT NaCl vs SCA NaCl, $p=0.0002 ;$ WT NaCl vs SCA DiAP, $p=0.14 ;$ SCA NaCl vs SCA DiAP, $p=0.03$ ). $d$, AP normalizes the latency to fall from an accelerated rotarod of 5-week-old SCA1 mice but has no significant effect on 5-week-old control mice (WT NaCl, $n=24$; WT AP, $n=23 ; \mathrm{SCA} \mathrm{NaCl}, n=24 ;$ SCA DiAP, $n=24$; two-way ANOVA followed by Fisher's test, WT NaCl vs WT AP, $p=0.14 ;$ WT NaCl vs SCA NaCl, $p=0.0035 ;$ WT NaCl vs SCA AP, $p=0.16 ;$ SCA NaCl vs SCA AP, $p=0.04)$. $e$, Continuous chronic treatment with DiAP using subcutaneous osmotic pumps improves motor performance of 5 -week-0ld SCA1 mice [WT NaCl $(n=20)$, SCA NaCl $(n=19)$, $\operatorname{SCA}$ DiAP $(n=19)$, two-way ANOVA followed by Fisher's test, WT NaCl vs SCA NaCl, $p=2 \times 10^{-5}$; WT NaCl vs SCA DiAP, $p=0.018 ;$ SCA NaCl vs SCA DiAP, $\left.p=0.035\right] . f$, Acute treatment with DiAP has no effect on the latency to fall from an accelerated rotarod of 18-week-old SCA1 mice (WT NaCl, $n=13 ;$ WT DiAP, $n=11 ;$ SCA NaCl, $n=20 ;$ SCA DiAP, $n=20 ;$ WT NaCl vs WT DiAP, $p=0.11$; WT NaCl vs SCA NaCl, $p=0.00004$; WT NaCl vs SCA DiAP, $p=7 \times 10^{-7}$; SCA NaCl vs SCA DiAP, $\left.p=0.21\right)$. $\boldsymbol{g}$, Acute treatment with DiAP has no effect on the grip strength of 18 -week-0ld SCA1 mice (one-way ANOVA followed by Fisher's test, WT NaCl vs WT DiAP, $p=0.43$; WT NaCl vs SCA NaCl, $p=1 \times 10^{-10}$; WT NaCl vs SCA DiAP, $p=2 \times 10^{-9} ;$ SCA NaCl vs SCA DiAP, $\left.p=0.49\right)$. $\boldsymbol{h}$, Acute treatment with DiAP has no effect on the latency to fall from a stationary thin rod of 18 -week-old SCA1 mice (one-way ANOVA followed by Fisher's test, WT NaCl vs WT DiAP, $p=0.3 ;$ WT NaCl vs SCA $\mathrm{NaCl}, p=3 \times 10^{-5} ;$ WT NaCl vs SCA DiAP, $\left.p=3 \times 10^{-6} ; \mathrm{SCA} \mathrm{NaCl} \mathrm{vs} \mathrm{SCA} \mathrm{DiAP,} p=0.07\right) . i$, Firing frequency of PCs from 18-week-old wild-type or SCA1 mice acutely treated with DiAP or saline (WT NaCl, $n=9 ;$ WT DiAP, $n=10 ;$ SCA NaCl, $n=10 ;$ SCA DiAP, $n=15 ;$ WTNaCl vs WTDiAP, $p=0.02 ;$ WTNaCl vs SCANaCl, $p=0.0003 ;$ WTNaCl vs SCADiAP, $p=0.19 ;$ SCANaCl vs SCADiAP, $p=$ 0.004).j, Typical firing pattern of Purkinje cells in cerebellar slice from 18-week-old WT and SCA1 mice after acute treatment with saline or DiAP. $\boldsymbol{k}$, Chronic treatment with DiAP increased the latency to fall from an accelerated rotarod of 18-week-old SCA1 mice (WT NaCl, $n=20$; WT DiAP, $n=12 ; \mathrm{SCA} \mathrm{NaCl}, n=18 ; \mathrm{SCA}$ DiAP, $n=19 ;$ WT NaCl vs WT DiAP, $p=0.68 ;$ WT NaCl vs SCA NaCl, $p=$ $3 \times 10^{-6}$; WT NaCl vs SCA DiAP, $p=0.004$; SCA NaCl vs SCA DiAP, $p=0.04$ ). I, Chronic treatment with DiAP increased the grip strength of 18-week-0ld SCA1 mice (WT NaCl vs WT DiAP, $p=0.85$; WT NaCl vs SCA NaCl, $p=0.0001$; WT NaCl vs SCA DiAP, $p=0.04$; SCA NaCl vs SCA DiAP, $p=0.04)$. $\boldsymbol{m}$, Chronic treatment with DiAP increased the latency to fall from a stationary thin rod of 18-week-old SCA1 mice (WT NaCl vs WT DiAP, $p=0.64$; WT NaCl vs SCA NaCl, $p=3 \times 10^{-5}$; WT NaCl vs SCA DiAP, $p=0.02 ;$ SCA NaCl vs SCA DiAP, $p=0.04$ ). $n$, Firing frequency of PCs from 18-week-old wild-type or SCA1 mice chronically treated with DiAP or saline (WT NaCl, $n=11 ;$ WT DiAP, $n=8 ;$ SCA NaCl, $n=22 ;$ SCA DiAP, $n=19 ;$ WT NaCl vs WT DiAP, $p=0.11 ;$ WT NaCl vs SCA $\mathrm{NaCl}, p=0.004 ;$ WT NaCl vs SCA DiAP, $p=0.25 ;$ SCA NaCl vs SCA DiAP, $p=0.03$ ). Error bars indicate SEM. $\boldsymbol{o}$, Typical firing pattern of Purkinje cells in cerebellar slice from 18 -week-0ld WT and SCA1 mice after chronic treatment with saline or DiAP. ${ }^{*} p<0.05 ;{ }^{* *} p<0.01 ;{ }^{* *} p<0.001$.

growth factors [like insulin-like growth factor 1 (Gatchel et al., 2008)] are involved in the protection provided by aminopyridines against PC atrophy. We propose that restoration of a normal firing frequency of PCs improves neurological deficits by increasing the production of growth factors such as BDNF.

Our study also shows that aminopyridines increase PCs spontaneous firing frequency in vivo. While it was "assumed" that aminopyridines increased PCs excitability (Strupp et al., 2008), the previous experimental evidence was provided by bath application of aminopyridines and intracellular recordings of PCs in acute slices after depolarization to membrane potentials that may not be physiological (Etzion and Grossman, 2001). It was recently reported that 4 -aminopyridine acted in a mouse model of epi- sodic ataxia type 2 by restoring the diminished precision of pacemaking in PCs (Alviña and Khodakhah, 2010). Our in vivo and slice recordings of PCs in SCA1 mice show a reduction in the firing rate without impairment of the regularity of their firing, so altered precision of pacemaking is not the only dysfunction of PCs leading to ataxia. The reduced firing frequency of PCs is expected to increase the firing frequency of deep cerebellar nuclei that is sufficient to cause ataxia (Shakkottai et al., 2004). A reduced firing frequency of PCs is also predicted in SCA13, caused by mutations of Kv3.3 channels (Waters et al., 2006). Contrary to the observation of Alviña and Khodakhah (2010) that bath application of "therapeutic concentration" of 4-AP does not increase the inhibitory drive of PCs, we show that acute and chronic treat- 
a pre 4AP
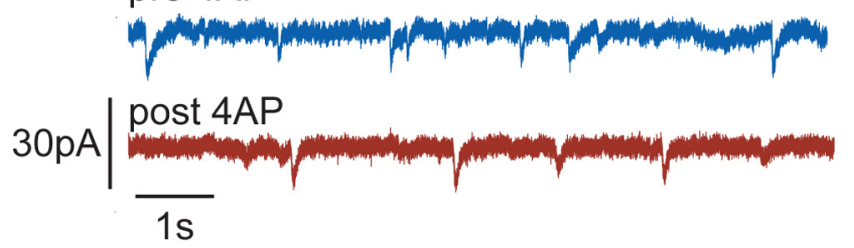

b

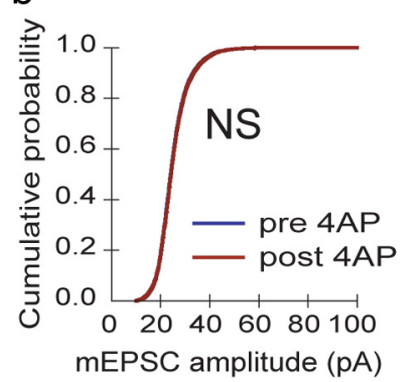

C

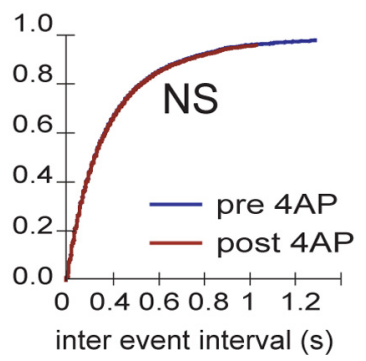

Figure 8. Bath application of 4-aminopyridine has no effect on the amplitude and frequency of $m E P S C s$ recorded in PCs from 4- to 5-week-old WT mice. $\boldsymbol{a}$, Typical mEPSCs recording before and 5 min after bath application of $50 \mu \mathrm{m}$ 4-aminopyridine. $\boldsymbol{b}$, Cumulative mean amplitude distributions of mEPSCS in PCs $(n=6)$ from mice before and after application of 4-aminopyridine (Kolmogorov-Smirnov test, $p>0.05$ ). c, Cumulative interevent time interval distributions of mEPSCS in PCS $(n=6)$ from mice before and after application of 4-aminopyridine (Kolmogorov-Smirnov test, $p>0.05$ ).

ments of mice with aminopyridines result in an increase in the firing rate of PCs.

As aminopyridines can potentially increase neuronal excitability via several mechanisms such as increase glutamate and/or GABA release, and as we administered aminopyridines systemically, our study has some limitations in the identification of the precise molecular mechanism by which aminopyridines increase the firing rate of PCs in vivo. We cannot rule out an action of aminopyridines in different brain regions and an indirect effect on the firing rate of PCs in the cerebellum. Kv4.3 channels expressed in Purkinje cells are possible targets, but we cannot not rule out an action on other channels. For example, Wu et al. (2009) described an unexpected activation of high voltageactivated calcium channels by aminopyridines in acutely dissociated neurons. Such an activation in cerebellar Purkinje cells could also contribute to the increased firing rate and improvement of the phenotype of SCA1 mice. Acutely and chronically treated animals were carefully monitored and showed no manifestation of epilepsy (a side effect of aminopyridines that was obvious with much higher concentrations of these drugs in vivo). Acute treatment with aminopyridines improved the motor behavior of SCA1 but not WT mice. Chronic treatment with 3,4-DiAP increased BDNF levels in the cerebellum of SCA1 but not WT mice. Together, these data raise the possibility that PCs from SCA1 mice- that show a very low firing rate at early presymptomatic stages - could be especially sensitive to the increase of excitability caused by aminopyridines. The reduced internalization of Kv4.3 channels in PCs from SCA1 mice provides a possible mechanism for a sensitization of PCs from SCA1 mice to a direct effect of aminopyridines in vivo.

In the studied mouse model of SCA1, aminopyridines correct the early dysfunction characterized by reduced firing rate of PCs, alleviate early behavioral deficits, and are beneficial on later stages associated with cell atrophy. Aminopyridines, which have been used safely for decades in patients with Lambert-Eaton syndrome and multiple sclerosis (McEvoy et al., 1989; Judge and Bever, 2006), which are effective in some cerebellar disorders such as episodic ataxia type 2 and downbeat nystagmus (Strupp et al., 2003, 2004, 2008), and which recently showed a modest symptomatic benefit in SCA6 (Tsunemi et al., 2010), could also have symptomatic or neuroprotective effects in patients with SCA1, an incurable progressive disease leading to severe disability and death.

\section{References}

Adcock KH, Metzger F, Kapfhammer JP (2004) Purkinje cell dendritic tree development in the absence of excitatory neurotransmission and of brain-derived neurotrophic factor in organotypic slice cultures. Neuroscience 127:137-145.

Alviña K, Khodakhah K (2010) The therapeutic mode of action of 4-aminopyridine in cerebellar ataxia. J Neurosci 30:7258-7268.

Balkowiec A, Katz DM (2002) Cellular mechanisms regulating activitydependent release of native brain-derived neurotrophic factor from hippocampal neurons. J Neurosci 22:10399-10407.

Beazely MA, Lim A, Li H, Trepanier C, Chen X, Sidhu B, Macdonald JF (2009) Platelet-derived growth factor selectively inhibits NR2B-containing $\mathrm{N}$-methyl-D-aspartate receptors in CA1 hippocampal neurons. J Biol Chem 284:8054-8063.

Burright EN, Clark HB, Servadio A, Matilla T, Feddersen RM, Yunis WS, Duvick LA, Zoghbi HY, Orr HT (1995) SCA1 transgenic mice: a model for neurodegeneration caused by an expanded CAG trinucleotide repeat. Cell 82:937-948.

Carter AR, Chen C, Schwartz PM, Segal RA (2002) Brain-derived neurotrophic factor modulates cerebellar plasticity and synaptic ultrastructure. J Neurosci 22:1316-1327.

Clark HB, Burright EN, Yunis WS, Larson S, Wilcox C, Hartman B, Matilla A, Zoghbi HY, Orr HT (1997) Purkinje cell expression of a mutant allele of SCA1 in transgenic mice leads to disparate effects on motor behaviors, followed by a progressive cerebellar dysfunction and histological alterations. J Neurosci 17:7385-7395.

Dieni S, Rees S (2002) Distribution of brain-derived neurotrophic factor and $\operatorname{TrkB}$ receptor proteins in the fetal and postnatal hippocampus and cerebellum of the guinea pig. J Comp Neurol 454:229-240.

Etzion Y, Grossman Y (2001) Highly 4-aminopyridine sensitive delayed rectifier current modulates the excitability of guinea pig cerebellar Purkinje cells. Exp Brain Res 139:419-425.

Furutani K, Okubo Y, Kakizawa S, Ino M (2006) Postsynaptic inositol 1,4,5-trisphosphate signaling maintains presynaptic function of parallel fiber-Purkinje cell synapses via BDNF. Proc Natl Acad Sci U S A 103:8528-8533.

Gatchel JR, Watase K, Thaller C, Carson JP, Jafar-Nejad P, Shaw C, Zu T, Orr HT, Zoghbi HY (2008) The insulin-like growth factor pathway is altered in spinocerebellar ataxia type 1 and type 7. Proc Natl Acad Sci U S A 105:1291-1296.

Green JT, Steinmetz JE (2005) Purkinje cell activity in the cerebellar anterior lobe after rabbit eyeblink conditioning. Learn Mem 12:260-269.

Hammond RS, Lin L, Sidorov MS, Wikenheiser AM, Hoffman DA (2008) Protein kinase a mediates activity-dependent Kv4.2 channel trafficking. J Neurosci 28:7513-7519.

Hisatsune C, Kuroda Y, Akagi T, Torashima T, Hirai H, Hashikawa T, Inoue T, Mikoshiba K (2006) Inositol 1,4,5-trisphosphate receptor type 1 in granule cells, not in Purkinje cells, regulates the dendritic morphology of Purkinje cells through brain-derived neurotrophic factor production. J Neurosci 26:10916-10924.

Hounsgaard J, Midtgaard J (1988) Intrinsic determinants of firing pattern in Purkinje cells of the turtle cerebellum in vitro. J Physiol 402:731-749.

Inoue T, Lin X, Kohlmeier KA, Orr HT, Zoghbi HY, Ross WN (2001) Calcium dynamics and electrophysiological properties of cerebellar Purkinje cells in SCA1 transgenic mice. J Neurophysiol 85:1750-1760.

Judge SI, Bever CT Jr (2006) Potassium channel blockers in multiple sclerosis: neuronal $\mathrm{Kv}$ channels and effects of symptomatic treatment. Pharmacol Ther 111:224-259.

Kashiwabuchi N, Ikeda K, Araki K, Hirano T, Shibuki K, Takayama C, Inoue Y, Kutsuwada T, Yagi T, Kang Y (1995) Impairment of motor coordination, Purkinje cell synapse formation, and cerebellar long-term depression in GluR delta 2 mutant mice. Cell 81:245-252.

Kim J, Jung SC, Clemens AM, Petralia RS, Hoffman DA (2007) Regulation of dendritic excitability by activity-dependent trafficking of the 

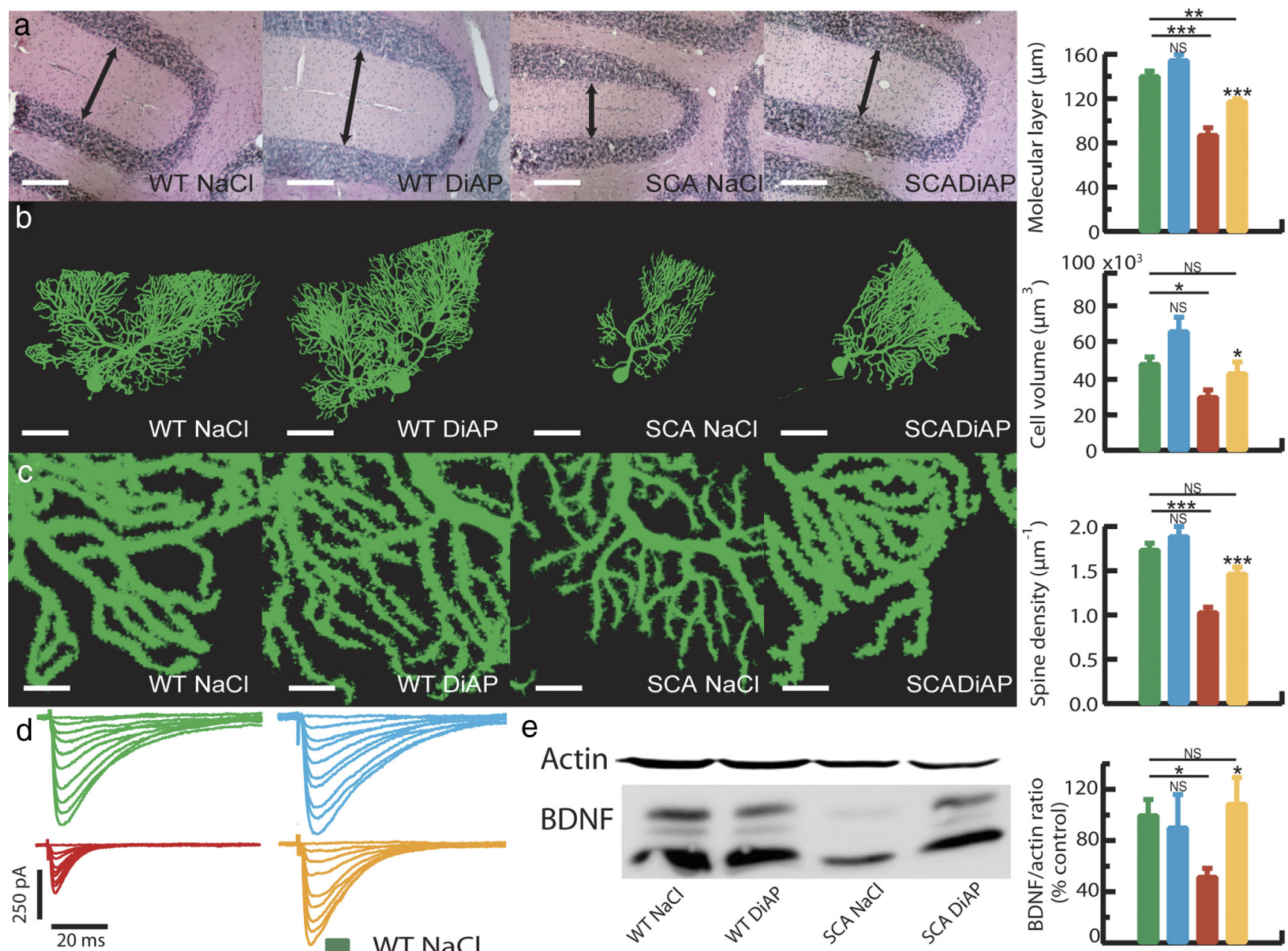

e

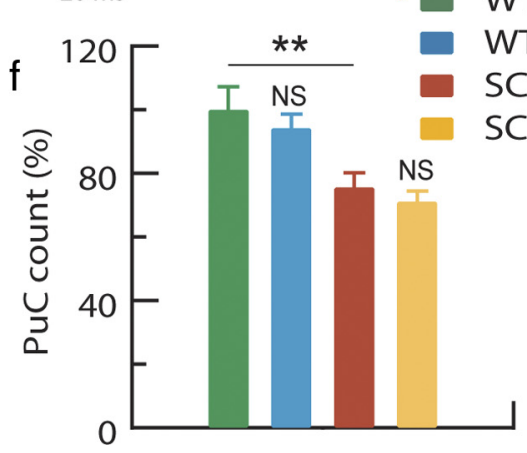

WT $\mathrm{NaCl}$

Actin

BDNF
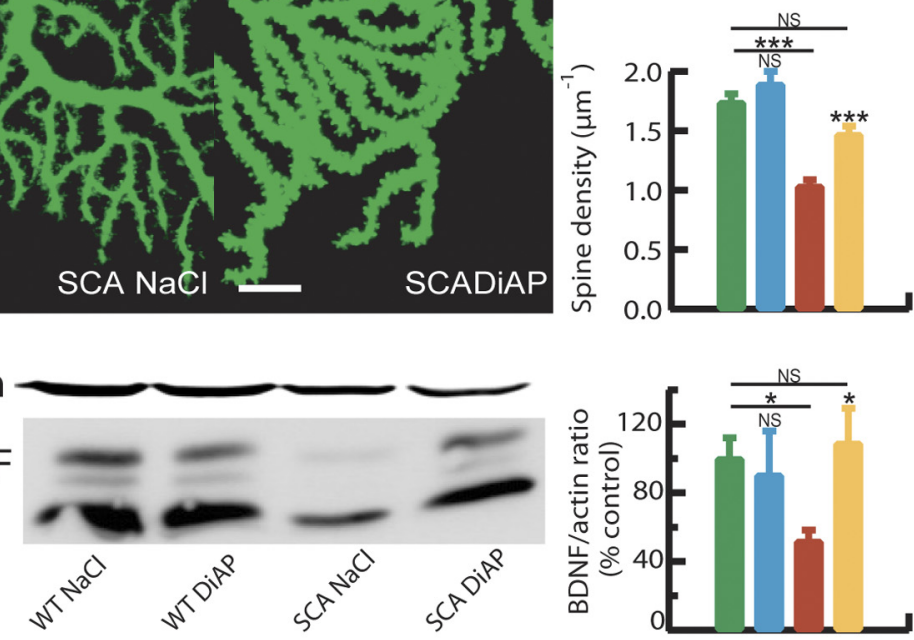

WT DiAP

CA NaCl 9

h
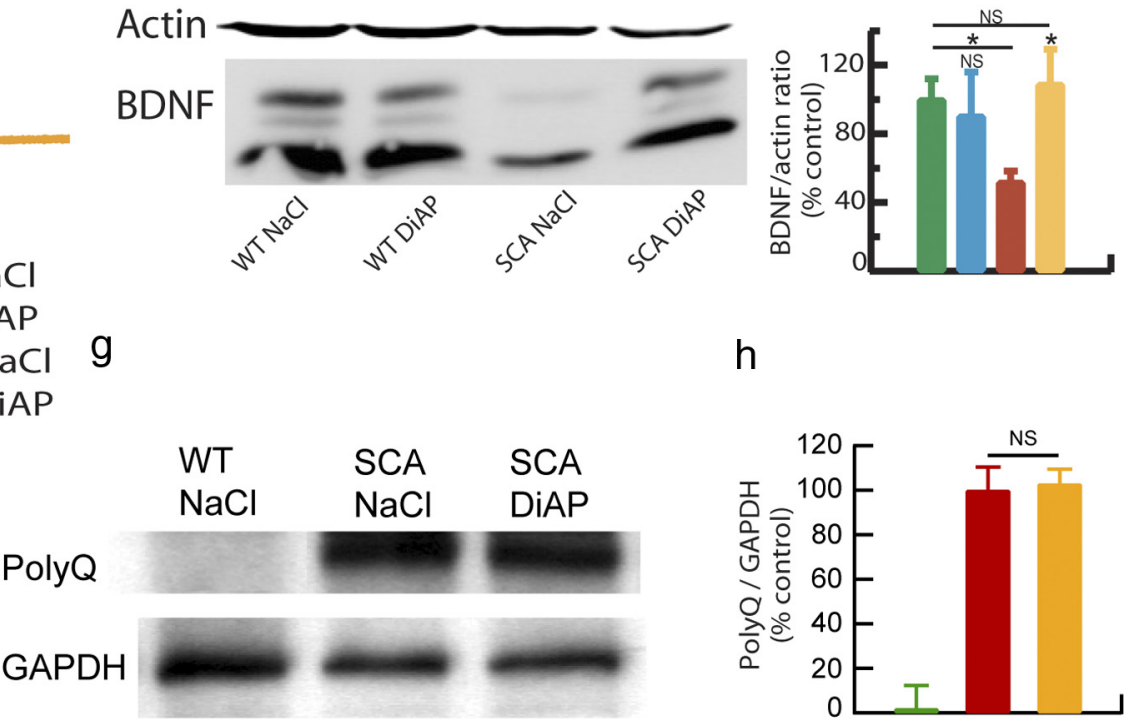

Figure 9. Chronic treatment with $I K_{A}$ blockers partially protects $\mathrm{PC}$ s from atrophy and increases cerebellar levels of BDNF in SCA1 mice. $\boldsymbol{a}$, Typical appearance and quantification of the thickness of the molecular layer of 18-week-old WT or SCA1 mice chronically treated with NaCl or DiAP (WT NaCl, $n=6$ mice; WT DiAP, $n=4 ;$ SCA NaCl, $n=5 ;$ SCA DiAP, $n=7$; one-way ANOVA followed by Fisher's test, WT NaCl vs WT DiAP, $p=0.06$; WT NaCl vs SCA NaCl, $p=9 \times 10^{-8}$; WT NaCl vs SCA DiAP, $p=0.0015$; SCA NaCl vs SCA DiAP, $\left.p=2 \times 10^{-6}\right)$. $\boldsymbol{b}$, Typical appearance and quantification of the volume of biocytin-loaded PCs from 18-week-old WT or SCA1 mice chronically treated with NaCl or DIAP (WT NaCl, $n=10$ cells; WT DiAP, $n=13 ;$ SCA NaCl, $n=32 ; S C A$ DiAP, $n=18$; one-way ANOVA followed by Fisher's test, WT NaCl vs WT DiAP, $p=0.07$; WT NaCl vs SCA NaCl, $p=0.037$; WT NaCl vs SCA DiAP, $p=0.58 ;$ SCA NaCl vs SCA DiAP, $p=0.04)$. c, Typical appearance of the dendritic tree of PCs from 18-week-old WT or SCA1 mice chronically treated with NaCl or DIAP and quantification of the density of dendritic spines (2 dendrites analyzed per cell; one-way ANOVA followed by Fisher's test, WT NaCl vs WT DiAP, $p=0.33$; WT NaCl vs SCA NaCl, $p=2 \times 10^{-5}$; WT NaCl vs SCA DiAP, $p=0.12 ;$ SCA NaCl vs SCA DiAP, $\left.p=0.0006\right) . d$, Representative recordings obtained from PCs in response to an increasing stimulation of parallel fibers from 18-week-old WT or SCA1 mice chronically treated with NaCl or DiAP. e, Western blotting for BDNF and actin from cerebella of 18-week-old WT or SCA1 mice chronically treated with NaCl or DiAP and quantification of BDNF/actin ratio (WT NaCl, $n=8 ;$ WT DiAP, $n=4 ; S C A N a C l, n=8 ; S C A$ DiAP, $n=$ 8; one-way ANOVA followed by Fisher's test, WT NaCl vs WT DiAP, $p=0.7$; WT NaCl vs SCA NaCl, $p=0.029 ;$ WT NaCl vs SCA DiAP, $p=0.67 ;$ SCA NaCl vs SCA DiAP, $p=0.011) . f$, Normalized Purkinje cell count (11 WT NaCl, 4 WT DiAP, 9SCA NaCl, 13 SCA DiAP; one-way ANOVA followed by Fisher's test, WT NaCl vs WT DiAP, $p=0.19 ;$ WT NaCl vs SCA NaCl, $p=0.003$; WT NaCl vs SCA DiAP, $p=$ 0.0001 ; SCA NaCl vs SCA DiAP, $p=0.54$ ). $\boldsymbol{g}$, Representative Western blot of ataxin-1 (top) and a loading control (GAPDH) in cerebella from saline-treated WT mice (first lane), saline-treated SCA1 mice (second lane), and DiAP-treated SCA1 mice (last lane). $\boldsymbol{h}$, Normalized ataxin-1/GAPDH ratio ( $n=4 /$ group; $100 \%=$ saline-treated SCA1 mice, SCA NaCl vs SCA DiAP, $p=0.82$ ) showed no change in expanded ataxin-1 expression by chronic treatment with 3.4 diaminopyridine. Error bars indicate SEM. ${ }^{*} p<0.05 ;{ }^{* *} p<0.01 ;{ }^{* * *} p<0.001$.

A-type $\mathrm{K}^{+}$channel subunit Kv4.2 in hippocampal neurons. Neuron 54:933-947.

Klee R, Ficker E, Heinemann U (1995) Comparison of voltagedependent potassium currents in rat pyramidal neurons acutely iso- lated from hippocampal regions CA1 and CA3. J Neurophysiol 74:1982-1995

Lei Z, Deng P, Xu ZC (2008) Regulation of Kv4.2 channels by glutamate in cultured hippocampal neurons. J Neurochem 106:182-192. 
Lin X, Antalffy B, Kang D, Orr HT, Zoghbi HY (2000) Polyglutamine expansion down-regulates specific neuronal genes before pathologic changes in SCA1. Nat Neurosci 3:157-163.

McAllister AK, Katz LC, Lo DC (1996) Neurotrophin regulation of cortical dendritic growth requires activity. Neuron 17:1057-1064.

McEvoy KM, Windebank AJ, Daube JR, Low PA (1989) 3,4-Diaminopyridine in the treatment of Lambert-Eaton myasthenic syndrome. N Engl J Med 321:1567-1571.

Milnerwood AJ, Gladding CM, Pouladi MA, Kaufman AM, Hines RM, Boyd JD, Ko RW, Vasuta OC, Graham RK, Hayden MR, Murphy TH, Raymond LA (2010) Early increase in extrasynaptic NMDA receptor signaling and expression contributes to phenotype onset in Huntington's disease mice. Neuron 65:178-190.

Ohira K, Funatsu N, Nakamura S, Hayashi M (2004) Expression of BDNF and TrkB receptor subtypes in the postnatal developing Purkinje cells of monkey cerebellum. Gene Expr Patterns 4:257-261.

Rivera JF, Ahmad S, Quick MW, Liman ER, Arnold DB (2003) An evolutionarily conserved dileucine motif in Shal $\mathrm{K}^{+}$channels mediates dendritic targeting. Nat Neurosci 6:243-250.

Sacco T, Tempia F (2002) A-type potassium currents active at subthreshold potentials in mouse cerebellar Purkinje cells. J Physiol 543:505-520.

Schiffmann SN, Cheron G, Lohof A, d'Alcantara P, Meyer M, Parmentier M, Schurmans S (1999) Impaired motor coordination and Purkinje cell excitability in mice lacking calretinin. Proc Natl Acad Sci U S A 96:5257-5262.

Schilling K, Dickinson MH, Connor JA, Morgan JI (1991) Electrical activity in cerebellar cultures determines Purkinje cell dendritic growth patterns. Neuron 7:891-902.

Serôdio P, Rudy B (1998) Differential expression of Kv4 K ${ }^{+}$channel subunits mediating subthreshold transient $\mathrm{K}^{+}$(A-type) currents in rat brain. J Neurophysiol 79:1081-1091.

Serra HG, Byam CE, Lande JD, Tousey SK, Zoghbi HY, Orr HT (2004) Gene profiling links SCA1 pathophysiology to glutamate signaling in Purkinje cells of transgenic mice. Hum Mol Genet 13:2535-2543.

Servais L, Hourez R, Bearzatto B, Gall D, Schiffmann SN, Cheron G (2007) Purkinje cell dysfunction and alteration of long-term synaptic plasticity in fetal alcohol syndrome. Proc Natl Acad Sci U S A 104:9858-9863.

Shakkottai VG, Chou CH, Oddo S, Sailer CA, Knaus HG, Gutman GA, Barish ME, LaFerla FM, Chandy KG (2004) Enhanced neuronal excitability in the absence of neurodegeneration induces cerebellar ataxia. J Clin Invest 113:582-590.

Strupp M, Schüler O, Krafczyk S, Jahn K, Schautzer F, Büttner U, Brandt T (2003) Treatment of downbeat nystagmus with 3,4-diaminopyridine: a placebo-controlled study. Neurology 61:165-170.

Strupp M, Kalla R, Dichgans M, Freilinger T, Glasauer S, Brandt T (2004) Treatment of episodic ataxia type 2 with the potassium channel blocker 4-aminopyridine. Neurology 62:1623-1625.

Strupp M, Kalla R, Glasauer S, Wagner J, Hüfner K, Jahn K, Brandt T (2008) Aminopyridines for the treatment of cerebellar and ocular motor disorders. Prog Brain Res 171:535-541.

Tobin AJ, Signer ER (2000) Huntington's disease: the challenge for cell biologists. Trends Cell Biol 10:531-536.

Tsunemi T, Ishikawa K, Tsukui K, Sumi T, Kitamura K, Mizusawa H (2010) The effect of 3,4-diaminopyridine on the patients with hereditary pure cerebellar ataxia. J Neurol Sci 292:81-84.

van der Heyden MA, Wijnhoven TJ, Opthof T (2006) Molecular aspects of adrenergic modulation of the transient outward current. Cardiovasc Res $71: 430-442$.

Vandesompele J, De Preter K, Pattyn F, Poppe B, Van Roy N, De Paepe A, Speleman F (2002) Accurate normalization of real-time quantitative RT-PCR data by geometric averaging of multiple internal control genes. Genome Biol 3:RESEARCH0034.

Wang D, Schreurs BG (2006) Characteristics of IA currents in adult rabbit cerebellar Purkinje cells. Brain Res 1096:85-96.

Waters MF, Minassian NA, Stevanin G, Figueroa KP, Bannister JP, Nolte D, Mock AF, Evidente VG, Fee DB, Müller U, Dürr A, Brice A, Papazian DM, Pulst SM (2006) Mutations in voltage-gated potassium channel KCNC3 cause degenerative and developmental central nervous system phenotypes. Nat Genet 38:447-451.

Weisz CJ, Raike RS, Soria-Jasso LE, Hess EJ (2005) Potassium channel blockers inhibit the triggers of attacks in the calcium channel mouse mutant tottering. J Neurosci 25:4141-4145.

Wu ZZ, Li DP, Chen SR, Pan HL (2009) Aminopyridines potentiate synaptic and neuromuscular transmission by targeting the voltage-activated calcium channel beta subunit. J Biol Chem 284:36453-36461.

Zu T, Duvick LA, Kaytor MD, Berlinger MS, Zoghbi HY, Clark HB, Orr HT (2004) Recovery from polyglutamine-induced neurodegeneration in conditional SCA1 transgenic mice. J Neurosci 24:8853-8861. 